\title{
Impedance measurements of the Spallation Neutron Source extraction kicker system
}

\author{
H. Hahn \\ Brookhaven National Laboratory, Upton, New York 11973, USA
}

(Received 10 June 2004; published 19 October 2004)

\begin{abstract}
Transverse coupling impedance measurements of the Spallation Neutron Source (SNS) beam extraction system were performed and the results are here reported. The SNS beam extraction system is composed from 14 subsystems, each of which consists of a vertical kicker magnet plus a pulse forming network (PFN). Impedance bench measurements were performed on one large and one small aperture magnet, stand-alone as well as assembled with the first-article production PFN. The impedance measuring methods to cover the interesting frequency range from below 1 to $100 \mathrm{MHz}$ are described in considerable detail. The upper frequency range is properly covered by the conventional twin-wire method but it had to be supplemented at the low-frequency end by a direct input impedance measurement at the magnet busbar. Required modifications of the PFN to maintain the impedance budget are discussed. The total impedance estimate was finally obtained by quadratic scaling with vertical aperture from the two tested kicker subsystems.
\end{abstract}

DOI: $10.1103 /$ PhysRevSTAB.7.103501

PACS numbers: 29.27.Bd

\section{INTRODUCTION}

The driving terms of instabilities in accelerator/storage rings depend on the beam surroundings which are conveniently described by impedances. Establishing and maintaining a coupling impedance budget becomes an important part of designing a high current accelerator. Theoretical estimates for typical accelerator components have been developed and are available in the standard literature. For critical devices, the estimates need to be confirmed by impedance bench measurements. The basic concept of bench measurements relies on simulating the beam by a wire for longitudinal or a twin-wire Lecher line for transverse measurements. The measurements typically involve a measurement of the device under test (DUT) and of a reference structure with the difference or ratio of the data used to interpret the coupling impedance [1]. The theoretical basis and a method of transverse impedance measurements involving a single loop was reported in the seminal paper by Nassibian and Sacherer (NS) [2]. The by now standard method was first applied by Walling et al. [3] and involves the insertion of twin wires into the magnet aperture, respectively, the reference tube, and the measurement of the forward transmission scattering coefficients with a network analyzer. The various formulas for the interpretation of the data have been discussed at length in a recent paper [4].

In the case of the Spallation Neutron Source (SNS) accumulator ring [5], the extraction kicker represents the most critical impedance item and the prototype magnet was intensively studied using the twin-wire method [6-9]. The immediate impetus for the present study came from the desire to extend the low-frequency range and the requirement of an improved impedance match of the extraction system involving the kicker magnet connected to the pulse forming network (PFN) by the $\sim 200 \mathrm{~m}$ long cable connections. The frequency range of interest to SNS covers frequencies, from $\sim 100 \mathrm{MHz}$ down to below $1 \mathrm{MHz}$, with emphasis on the $1-10 \mathrm{MHz}$ range. Applying the wire method to the low-frequency part entails difficulties. Especially at the low-frequency end, at $\sim 1 \mathrm{MHz}$ and below, the signals become very weak and the noise and drift of the instrument lead to results which may no longer be valid. This provided the motivation to extend the study and to search for measurement methods beyond the obvious averaging and smoothing of the data. Modified measurement techniques using only the available instrument and equipment gave reliable impedance results down to a few $100 \mathrm{kHz}$ and are discussed in this paper. Extending the frequency range further down would have involved even greater effort. For example, the transverse impedance study of the Large Hadron Collider dump kicker down to a few $100 \mathrm{~Hz}$ was performed by Mostacci et al. with multiturn loops [10].

The low-frequency range is on one hand the cause of a problem, but on the other hand it also presents the possibility of treating the beam to magnet interaction as lumped element transformer. It was already pointed out by NS that the coupling impedance is generated by the flux coupled to the external system plus a contribution from image currents. At low frequencies, the impedance is dominated by the coupled flux impedance which can be described by the transformer model. The model consists of the magnet inductance, with the external impedance in parallel across the busbar terminals. The mechanical construction of the SNS extraction kicker busbar provides a short gap, where a direct measurement of the coupled impedance can be done. The justification of the direct method [11] and its limits are a central topic of the present paper. In this paper, three different approaches, that is the twin-wire measurement with external reference, the measurement with shorted busbar as reference, and the direct 
impedance measurements at the busbar, are described and compared.

The ultimate goal of the work presented here was to establish an impedance estimate of the total SNS extraction kicker system. The system consists of 14 subsystems each being composed of a kicker magnet plus its PFN connected by $\sim 200 \mathrm{~m}$ long cables. Although a substantial database for the stand-alone prototype kicker magnet was available from previous studies, the results in this paper represent new measurements. The extension to the lowfrequency end made it necessary to obtain continuously measured data over the entire frequency range of interest. Impedance measurements reported here were performed separately on the large-aperture prototype magnet and the smallest-aperture production magnet, K11. The subsystem impedance was then obtained with either magnet connected to the "first-article" production PFN. Furthermore, the measurement of the assembled system showed an additional PFN-generated impedance, and studies of the PFN input impedance became necessary. Modifications of the PFN required to maintain the impedance budget were explored and are discussed. The total SNS kicker system impedance estimate was finally obtained by quadratic scaling with vertical magnet aperture from the two tested kicker systems. Although dedicated to the specific SNS kicker magnet system, the detailed discussion of the alternate impedance measurement methods is expected to be of more general interest.

\section{TWIN-WIRE MEASUREMENTS}

The transverse coupling impedance of a kicker magnet can be measured on the bench by using the standard method in which a twin-wire (TW) Lecher line, simulating the beam, is inserted into the device under test. Using a network analyzer, the forward transmission coefficient $S_{21}^{\mathrm{DUT}}$ of the kicker is determined and compared with the $S_{21}^{\mathrm{REF}}$ obtained in a reference measurement. The theoretical reference for the measurement of the transverse coupling impedance is the free space. However, a "true" free space without radiation effects is difficult to arrange, and the use of a beam tube with the accelerator dimensions is considered mandatory.

The setup for a TW measurement of a lumped kicker magnet, such as the SNS extraction kicker, is symbolically represented by the equivalent circuit in Fig. 1. The magnet has an inductance $L_{K}$, the TW line has an inductance $L_{L}$, and a characteristic impedance $R_{C}$. The coupling of the magnet to the TW line is given by the coupling coefficient $\kappa$, and the transformer ratio by $n=$ $\Delta / h$ with $\Delta$ and $h$ the wire spacing and the magnet vertical aperture, respectively. As already discussed by NS, the total impedance of a lumped kicker magnet is composed of the coupled part, which is due to the external termination $Z_{g}$ plus an uncoupled part due to image currents, represented by the intrinsic impedance $L_{I}$. Both

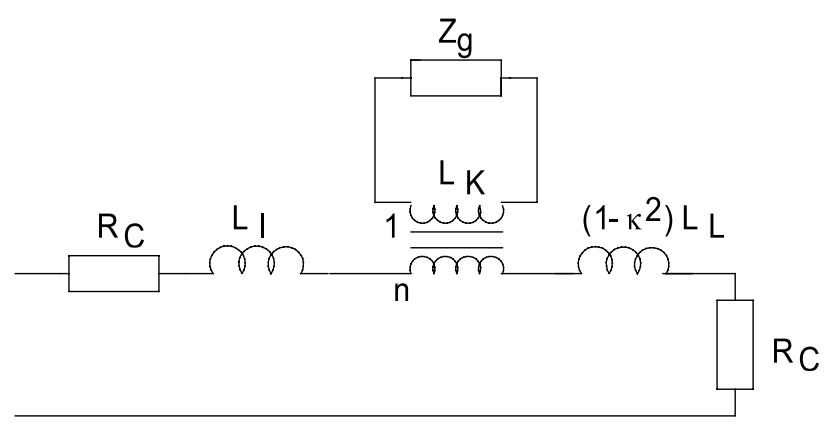

FIG. 1. Lumped kicker magnet circuit model.

the magnet and especially the intrinsic inductance are complex quantities to allow for ferrite losses. The "leakage" inductance $\kappa^{2} L_{L}$ leads to a difference between the impedance seen by the beam and measured by the TW method. In a typical case with the wire spacing small compared to the magnet aperture, this difference is inductive and can be neglected in the instability driving resistive term. In this vein, small stray inductance and capacitance values are not shown in the circuit, length differences between magnet and TW line are ignored, furthermore a small difference in inductance and impedance between the line when placed into the reference tube or in the magnet have been neglected.

The equivalent circuit suggests an alternate reference setup for the measurement of the coupled impedance part by shorting the magnet at the busbar gap. Errors due to mechanical changes or drift in the network analyzer are thus eliminated or at least reduced. The result for the termination generated resistive impedance component, which is of particular interest for the beam stability, remains unchanged, but the reactive part will differ from the result obtained with the reference tube. Note that the image current and leakage impedance is now part of the reference and is subtracted from the total impedance by this measurement. Furthermore, in the SNS kicker, the magnet geometry prevents a perfect short and the data requires a small correction and will be addressed below.

The scattering coefficients are interpreted according to the standard Hahn-Pedersen (HP) formula for lumped elements [4],

$$
Z^{\mathrm{DUT}}=2 R_{C}\left(S_{21}^{\mathrm{REF}} / S_{21}^{\mathrm{DUT}}-1\right),
$$

with $R_{C}$ the nominal impedance to which the TW line is matched, not necessarily the actual, line impedance. Primary matching to the instrument impedance, $R_{0}=$ $50 \Omega$, is done with transformers and completed with resistive matching. The characteristic line impedance is only approximately known, but mismatch errors are minimized and vanish at the low-frequency end by the use of the nominal $R_{C}$. The transverse coupling impedance now follows from the standard twin-wire measure- 
ment expression, as [2]

$$
Z_{\perp}=\frac{c}{\omega \Delta^{2}} Z^{\text {DUT }}
$$

with $\Delta$ the effective wire spacing to be discussed below. The transverse impedance has values in vertical and horizontal direction. The measurements in vertical, that is the kick direction, are here identified by $Z_{y}$ rather than the generic $Z_{\perp}$.

For the typical case in kicker magnets, one has $Z^{\text {DUT }} \ll R_{C}$, which leads to the alternative approximation for the transverse coupling impedance of the DUT, known as the log formula [3],

$$
Z_{\perp}=\frac{c Z^{\mathrm{DUT}}}{\omega \Delta^{2}} \approx 2 \frac{c R_{C}}{\omega \Delta^{2}} \ln \frac{S_{21}^{\mathrm{REF}}}{S_{21}^{\mathrm{DUT}}} .
$$

Unless noted differently, the results presented in this paper were obtained by using the conversion in the network analyzer corresponding to the standard lumped formula.

The scattering coefficients were measured with the network analyzer Agilent 8753ES, set for a linear frequency range from $30 \mathrm{kHz}$ to $10 \mathrm{MHz}$, respectively, to $100 \mathrm{MHz}$, with 1601 points. The preeminent, lowfrequency instrument errors of noise and drift were reduced by using a $100 \mathrm{~Hz}$ i.f. bandwidth and by limiting the time between measuring the DUT and the reference to minutes. Critical data were obtained by averaging over three sweeps. The data of a magnet locally impedance terminated can be corrected by smoothing; however, the PFN terminated magnet with its sharp resonances does not tolerate smoothing.

The accuracy of the resistive transverse coupling impedance is inherently noise limited at the low-frequency end by the $\omega^{-1}$ factor. The reactive part of the DUT impedance frequently is proportional to $\omega$ and thus is less sensitive to noise. The irreducible noise in the $S_{21}$ measurement is $\sim 10^{-4}$ peak corresponding to $>1 \mathrm{k} \Omega / \mathrm{m}$ at $1 \mathrm{MHz}$ with the TW line used here, clearly preventing low-frequency measurements.

\section{TWIN-WIRE LINE}

The impedance measurements were performed by using a twin-wire line, $126 \mathrm{~cm}$ long, homemade from $5 \times$ $7.5 \mathrm{~mm}$ rectangular tubes as shown in Fig. 2. The wire

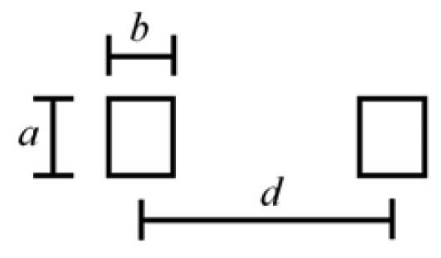

FIG. 2. The homemade twin-wire (TW) line. The dimensions are $a=7.5 \mathrm{~mm}, b=5 \mathrm{~mm}$, and $d=40.6 \mathrm{~mm}$. spacing is adjustable allowing changes of the inductance, characteristic impedance, and the signal level. Clearly, the signal increases with the wire spacing and the signalto-noise ratio is improved. The wire spacing was kept smaller than the half-height of the magnet aperture in order to remain in the perturbation regime, here $d \ll$ $12 \mathrm{~cm}$ for the prototype and $8 \mathrm{~cm}$ for the $\mathrm{K} 11$ magnet.

\section{A. Effective wire spacing}

The transverse impedance value from the interpretative Eqs. (2) and (3) depends quadratically on the effective wire spacing $\Delta$. Applying the theoretical results for round to rectangular wires, one finds the effective spacing to be

$$
\Delta=d \sqrt{1-(b / d)^{2}} .
$$

In the typical case of the wire size much smaller than the wire spacing, the effective $\Delta$ is given by the center-tocenter distance of the wires. This was checked by measuring the peak impedance value of the "open," i.e., unterminated, magnet, employing different TW lines [10]. Scaling of the peaks gave results which are consistent with the use of the simpler $\Delta \approx d$. For all measurements presented here an effective spacing of $\Delta=$ $40.6 \mathrm{~mm}$ will be used.

\section{B. Characteristic line impedance}

Interpretation of the measurements requires the knowledge of the characteristic impedance of the TW line. The characteristic impedance was measured directly with the communication signal analyzer Tektronix CSA 803 to be $Z_{L}=260 \pm 10 \Omega$.

The characteristic impedance has been estimated to be $Z_{L} \approx 277 \Omega$ from the theoretical expression for round wires by accommodating the rectangular lines with the use of $b^{\prime} \approx 2(a+b) / \pi$ instead of $b$ in [12]

$$
Z_{L}=\frac{c \mu_{0}}{\pi} \operatorname{acosh}\left(d / b^{\prime}\right) \text {. }
$$

An alternate determination of the characteristic impedance comes from the measurements of the line inductance per unit length. The TEM waves propagate on the TW line in air with the velocity of light, which implies the relation between characteristic impedance and inductance per unit length

$$
Z_{L}=c L_{L}^{\prime}
$$

The input impedance of the shorted TW line was measured in air at $1.5915 \mathrm{MHz}$ corresponding to the inductance of $L_{L}^{\prime} \approx 0.96 \mu \mathrm{H} / \mathrm{m}$, a value closer to $Z_{L} \approx 288 \Omega$.

\section{Impedance matching of TW line}

Although the bare TW line can be used without matching at the low-frequency end, $<10 \mathrm{MHz}$, to determine the basic kicker magnet properties, it is for most coupling 
impedance measurements necessary to match the characteristic line impedance to the $50 \Omega$ instrument impedance value. This can be done by resistive impedance matching or by the use of a transformer, or by a combination thereof. Commercial (North Hills) wideband transformers with a center-tapped secondary winding, serving as $180^{\circ}$ hybrid, were used for the measurements. Several transformers with different secondary impedance value and bandwidth were available and are identified according to their impedance as X300 (NH15880 with $100 \mathrm{kHz}-100 \mathrm{MHz}), \quad \mathrm{X} 200-100 \quad$ (NH15887 with $100 \mathrm{kHz}-100 \mathrm{MHz}$ ), and X200-60 (NH15888 with $10 \mathrm{kHz}-60 \mathrm{MHz}$ ).

Matching is confirmed by measuring the input impedance of the input transformer when the output transformer is terminated with the instrument impedance. The objective is a frequency-independent $50 \Omega$ input impedance. The input impedance of the twin line placed between the X300 and X200-100 transformers is shown in Fig. 3.

A flat response (blue curve) was achieved by matching the X200 transformer output impedance, $R_{0}=200 \Omega$, to the experimentally found value, $R_{C} \approx 250 \Omega$, the nominal characteristic impedance value to be used in the interpretative HP formula. Resistive matching is done on the input side with a parallel resistor $R_{P}$ and a series resistor $R_{\text {in }}$,
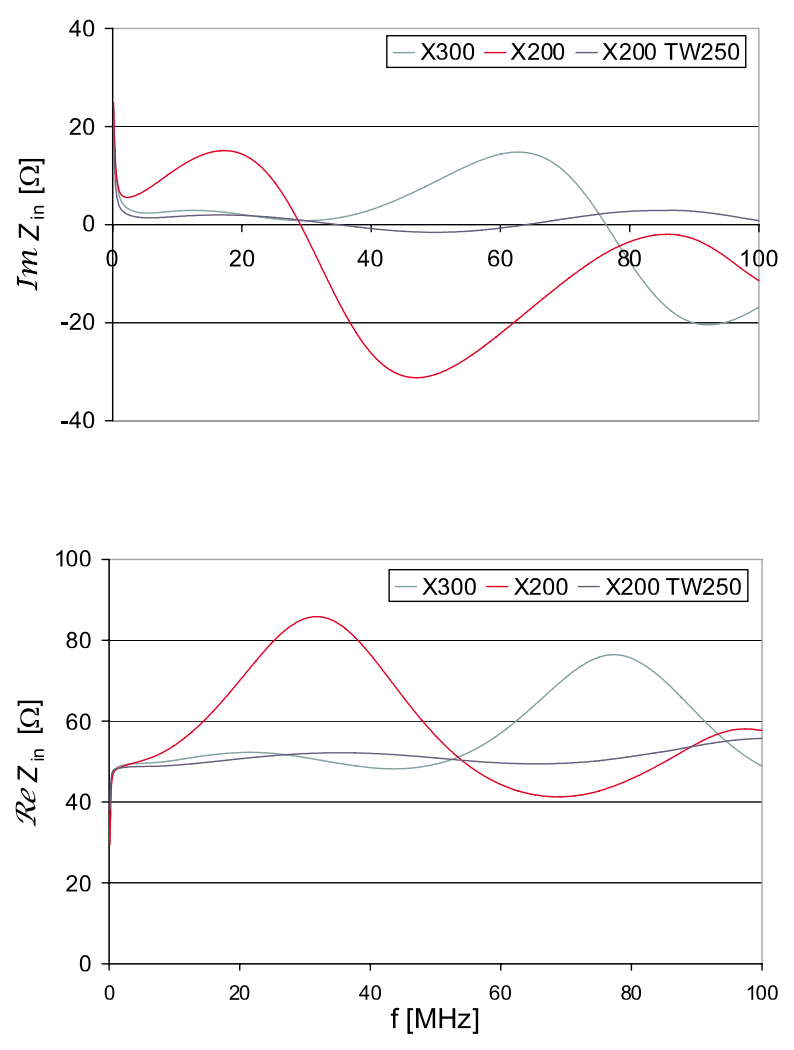

FIG. 3. (Color) Input impedance of TW-line with different transformers.

$$
\begin{aligned}
& R_{P}=R_{0} \sqrt{R_{C} /\left(R_{C}-R_{0}\right)} \approx 447 \Omega, \\
& R_{\mathrm{in}}=R_{C}-R_{0} R_{P} /\left(R_{0}+R_{P}\right)=2 \times 56 \Omega,
\end{aligned}
$$

and a series resistor on the output side, $R_{\text {out }}=R_{C}-R_{0}=2 \times 27 \Omega$.

The effect of the transformer bandwidth on the input impedance is shown in Fig. 4. Clearly, measurements below $\sim 60 \mathrm{MHz}$ are better done with the X200-60 transformers.

\section{Reference tube calibration}

The nonradiative impedance seen by an ultrarelativistic beam in free space is zero and is simulated by the current in the wire measurement. The use of a (perfectly conducting) reference tube for longitudinal wire measurements does not change the field distribution at the wire and yields the coupling impedance as seen by the beam. In contrast, the tube changes ("screens") the field at the twin wire during the reference measurement. The transverse TW measurements with a reference beam tube yield the incremental coupling impedance generated by replacing the beam tube with the device. Thus, the measured values differ from theoretical estimates which give the difference due to the ring component versus a zero impedance of the beam in free space.
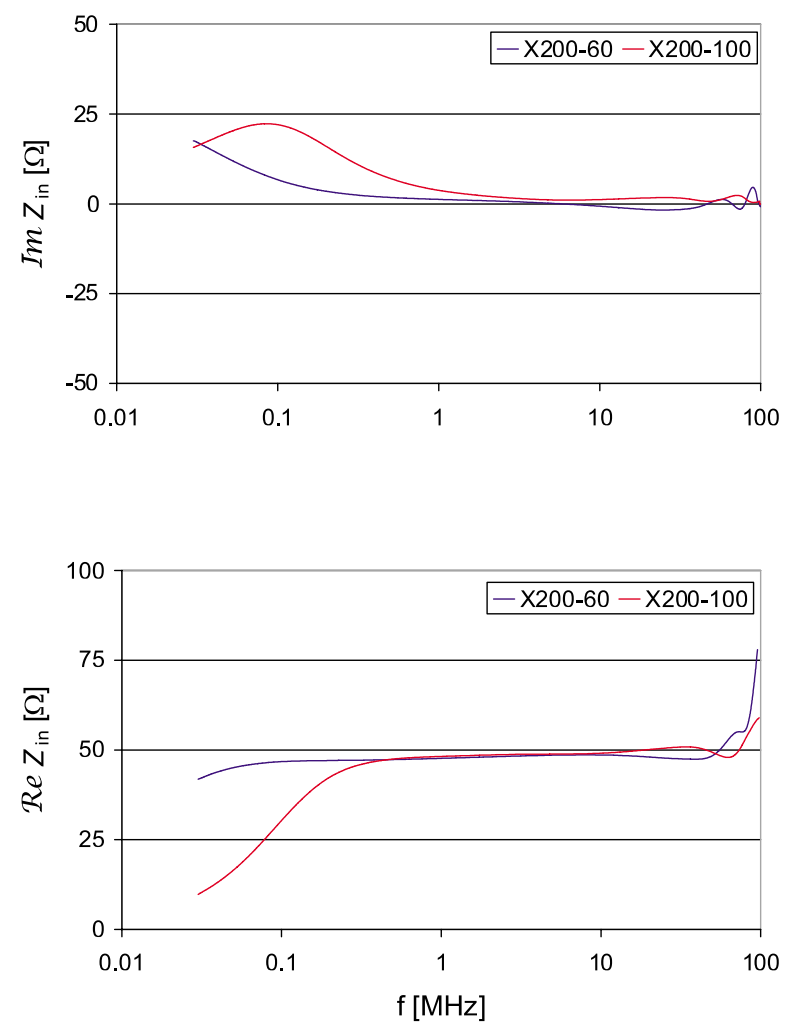

FIG. 4. (Color) Input impedance at transformers with different frequency range. 
The screening from placing the TW line into a "perfect" tube appears as a reduction of the line inductance (and characteristic impedance) given by [10]

$$
\Delta L \approx \frac{2 \mu_{0} \ell}{\pi}\left(\frac{\Delta}{D}\right)^{2} .
$$

For the reference tube with diameter $D=0.150 \mathrm{~m}$ and length $\ell=0.94 \mathrm{~m}$, one finds $\Delta L=0.05 \mu \mathrm{H}$, or scaled to the shorter magnet length, $\sim 0.02 \mu \mathrm{H}$, indeed comparable to measured data and instrumental measuring errors.

Although reactive and thus primarily of conceptual interest, the difference between the transverse impedance value from bench measurements in "free space" versus in a reference tube deserves consideration. In this vein, the reference beam tube was measured versus free space (air) and, in view of its length, was interpreted with the log formula

$$
Z^{\mathrm{DUT}}=-2 R_{C} \ln \frac{S_{21}^{\mathrm{TUBE}}}{S_{21}^{\mathrm{AIR}}} .
$$

Figure 5 shows the measured beam tube impedance versus free space. The impedance is due to the above screening effect plus the resistive wall impedance, both of which are expected to be monotonic with increasing frequency. At low frequencies, below $\sim 10 \mathrm{MHz}$, both
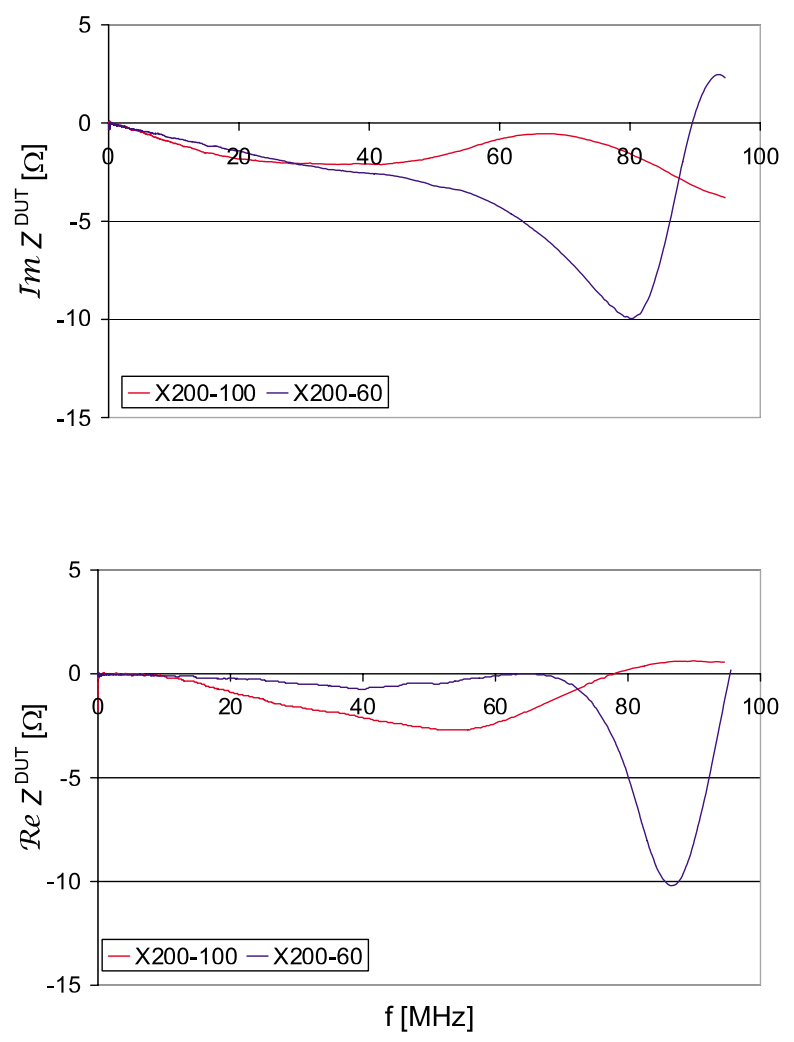

FIG. 5. (Color) Amplitude and phase of scattering coefficient in reference tube. transformers give the same resistive impedance and show the inductance change of $\sim 0.013 \mu \mathrm{H}$, somewhat lower than the theoretical estimate. At higher frequencies, the results in Fig. 5 are no longer monotonic with frequency and are attributed to the use of transformers with limited bandwidth. Furthermore, a true free space without radiation effects is difficult to arrange, and the use of a smooth beam tube with the accelerator dimensions is considered mandatory. The impedance data for the magnets, PFN, and the SNS system given below are all obtained with a beam tube as reference.

The reference scattering coefficient $S_{21}^{\mathrm{REF}}$ required for the interpretation of the wire measurements is obtained by placing the TW line with transformer assembly into the reference tube. The forward scattering coefficient of the assembly with either of the X200 transformers, matched to $250 \Omega$, was measured in the tube and is shown in Fig. 6. The result is in reasonable agreement with the nominal value $S_{21} \approx 0.55$. Measurements below $\sim 10 \mathrm{MHz}$ benefit from using the $60 \mathrm{MHz}$ transformer version, whereas the $100 \mathrm{MHz}$ version is better suited to the upper frequency range. However, since the SNS stability considerations focus on $\sim 4$ and $\sim 50 \mathrm{MHz}$, both transformers have been used for system measurements. The results are obtained from the $S_{21}$ ratio which largely eliminates the transformer properties.
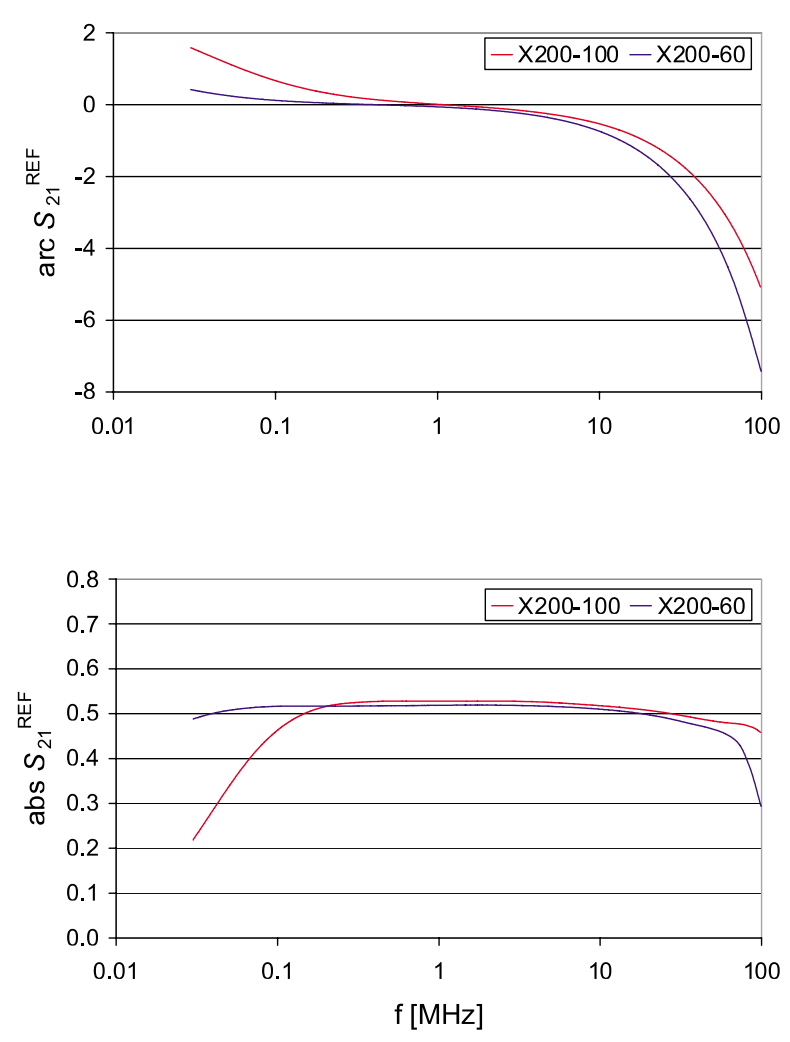

FIG. 6. (Color) Reference beam tube impedance versus free space. 


\section{SNS EXTRACTION KICKER MAGNET PROTOTYPE}

A full-size prototype of the SNS extraction kicker magnet served as the basis for the studies reported here. A schematic view of the freestanding window frame magnet as well as a picture of the magnet installed in its vacuum vessel is shown in Fig. 7. The vessel picture shows the busbar, its connection to the outside via the feedthrough, and the straps at the side connecting the upper and lower busbar plates. The busbar and ferrite blocks are fully isolated from the vessel by ceramic plates. So-called eddy current strips to reduce longitudinal impedance are also visible. The ferrite type is CMD5005 (Ceramic Magnetics Inc., Fairfield, NJ).

The kicker deflects vertically and has an aperture height of $h=24.8 \mathrm{~cm}$ between ferrites and $24.3 \mathrm{~cm}$ between the busbar. Its width and length dimensions are $w=15.9 \mathrm{~cm}, \ell=36 \mathrm{~cm}$, and the ferrite thickness is $t=$ $2.54 \mathrm{~cm}$. The busbar is $40 \mathrm{~cm}$ long, and the vacuum vessel has a $62 \mathrm{~cm}$ inner diameter and it is $88 \mathrm{~cm}$ long. Note the busbar end plates which represent a non-negligible capacity in the unconnected ("bare") magnet.

The electrical properties of the bare magnet are obtained from input impedance measurements at the busbar, using the $S_{11}$ function of the network analyzer. The instrument cable is connected via clip leads, which alone represent an inductance of $0.061 \mu \mathrm{H}$ and a capacity of $5.7 \mathrm{pF}$ which, since it is not part of the instrument calibration, needs to be subtracted from the measured data. The real and imaginary input impedance at the busbar is shown in Fig. 8 for the two cases of open and $25 \Omega$ termination.

The input impedance measurement yields accurate results over the entire frequency range of interest to the SNS stability considerations. The bare magnet has a low-frequency inductance of $L_{D}=1.015 \mu \mathrm{H}$ as measured and $L_{B}=0.954 \mu \mathrm{H}$ at the busbar with the clip leads subtracted. The $S_{11}$ measurement is accurate to a few percent and, for convenience sake, taken at $1.5915 \mathrm{MHz}$, the frequency quoted for most quoted val-
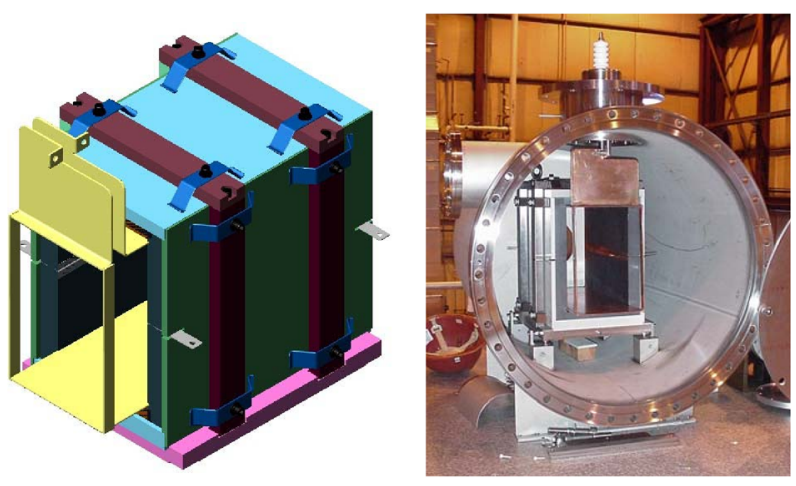

FIG. 7. (Color) SNS extraction kicker: schematic view (left), the kicker in the vessel (right).
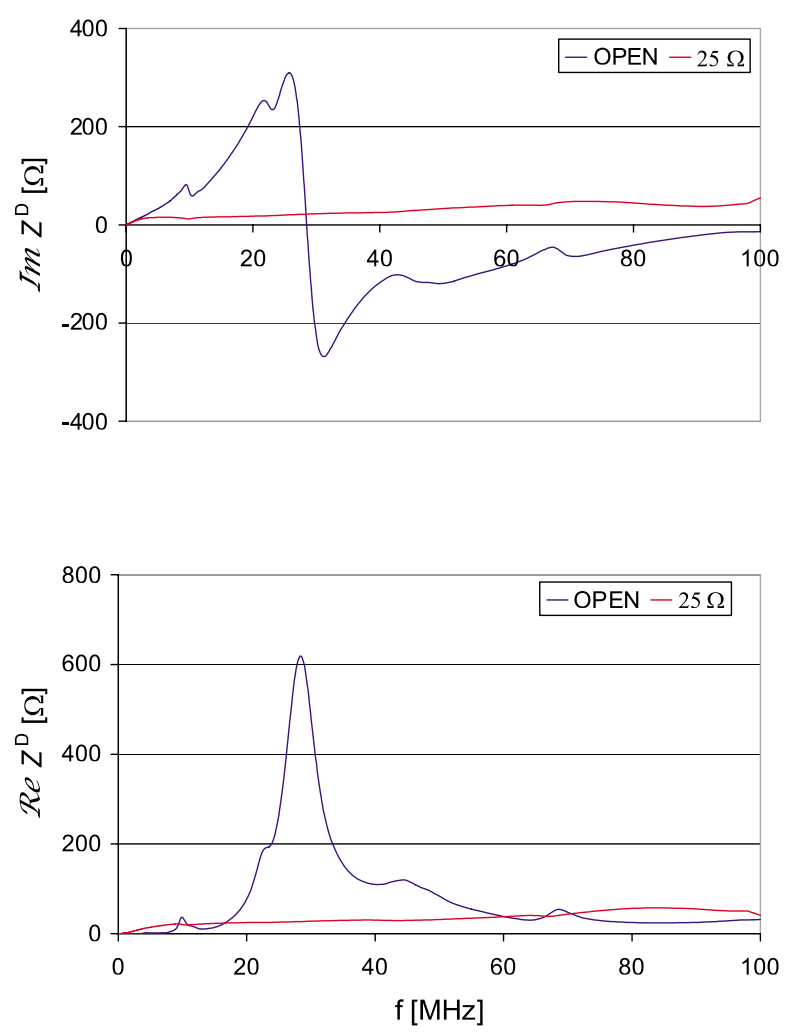

FIG. 8. (Color) Input impedance of the prototype kicker magnet at the busbar.

ues. The magnet geometry gives a nominal value for the inductance of $L_{B}>\mu_{0} h \ell / w=0.691 \mu \mathrm{H}$, without end effects and of $L_{B}<\mu_{0} h(\ell+w) / w=0.996$ with end effects estimated, a range covering the measured value. The estimate does not include the small inductance contribution $L_{S}$ attributable to the busbar side straps. Separating of inductance origins and identification of the strap inductance is required for the interpretation of the direct coupling impedance measurements, and will be discussed below. The resonance at $\sim 28 \mathrm{MHz}$ requires a capacitance of $\sim 33 \mathrm{pF}$ at the busbar terminal and is in part due to the busbar end plates themselves. Subtracting the capacity of the clip leads leaves the plate capacity at $C_{B} \approx 27 \mathrm{pF}$. The resonance of the bare magnet without measuring cable can now be estimated to shift from 28 to $\sim 32 \mathrm{MHz}$, the frequency expected in the TW measurements.

\section{Transformer properties of the kicker magnet}

Following the concept developed in the NS paper, the interaction of the beam with the kicker magnet is best analyzed by means of a model in which the twin-wire transmission line and the busbar are considered as a magnetic transformer. The line now represents the primary and the busbar the secondary of the transformer.

The transformer parameters are obtained from the full set of scattering coefficients in which the magnet is 
coupled to the twin-wire line by the mutual inductance. For this measurement, the TW line is shorted at one end. The magnet is disconnected from the feedthrough and the busbar is accessed with clip leads. The line in the magnet has a characteristic impedance, but in this setup needs not to be matched to the network analyzer impedance $R_{0}$. An input impedance measurement at the open line terminal yields the line inductance $L_{L}$, and the direct measurement at the busbar yields the magnet inductance $L_{D}$. The clip leads are here part of the setup and no correction is required.

The wire measurements are interpreted with regard to a simplified model derived from the standard electrical engineering equivalent circuit in Fig. 1. This model incorporates the impedance contributions from the coupled kicker inductance $L_{K}$, as well as that attributed to the leakage flux $\left(1-\kappa^{2}\right) L_{L}$. The mutual inductance $M$ and the coupling coefficient $\kappa$ are given by

$$
\kappa=\sqrt{\frac{M^{2}}{L_{K} L_{L}}},
$$

and the transformer ratio $n$ is given by the geometrical ratio of wire spacing and gap height in kick direction,

$$
n=\frac{M}{L_{K}}=\frac{\Delta}{h} \text {. }
$$

The transmission coefficient $S_{21}$ from the terminals of the line to the terminals of the busbar yields the mutual inductance via simple analytical steps. Based on the transformer model, and neglecting the presence of any stray capacitance, one can write the low-frequency expression for the scattering coefficient

$$
S_{21}=j \frac{2 \omega M R_{0}}{R_{0}^{2}+j \omega\left(L_{L}+L_{D}\right) R_{0}-\omega^{2}\left(L_{L} L_{D}-M^{2}\right)} .
$$

Note that $L_{D}$ represents the measured value at the busbar, which represents the sum of coupled inductance $L_{K}$ plus the side-strap inductance $L_{S}$. Finding the value of the side-strap inductance, which during operation actually is part of the magnet termination $Z_{g}$, becomes necessary to establish the base for further transverse impedance measurements.

Taylor expansion of Eq. (12) to first order in $\omega$ yields the expressions

$$
M \approx \frac{R_{0}}{2 \omega} \operatorname{abs}\left(S_{21}\right) \quad \text { and } \quad\left(L_{L}+L_{D}\right) \approx \frac{R_{0}}{\omega} \operatorname{arc}\left(S_{21}\right) .
$$

The low-frequency values are taken from Fig. 9 to be $M=0.134 \mu \mathrm{H}$. The second quantity is in fact a redundant result $\left(L_{L}+L_{D}\right)=2.494 \mu \mathrm{H}$, and is in reasonable agreement with the directly measured $L_{L}=1.272 \mu \mathrm{H}$ plus $L_{D}=1.015 \mu \mathrm{H}$. The coupled magnet inductance is now found from the mutual inductance to be $L_{K}=$
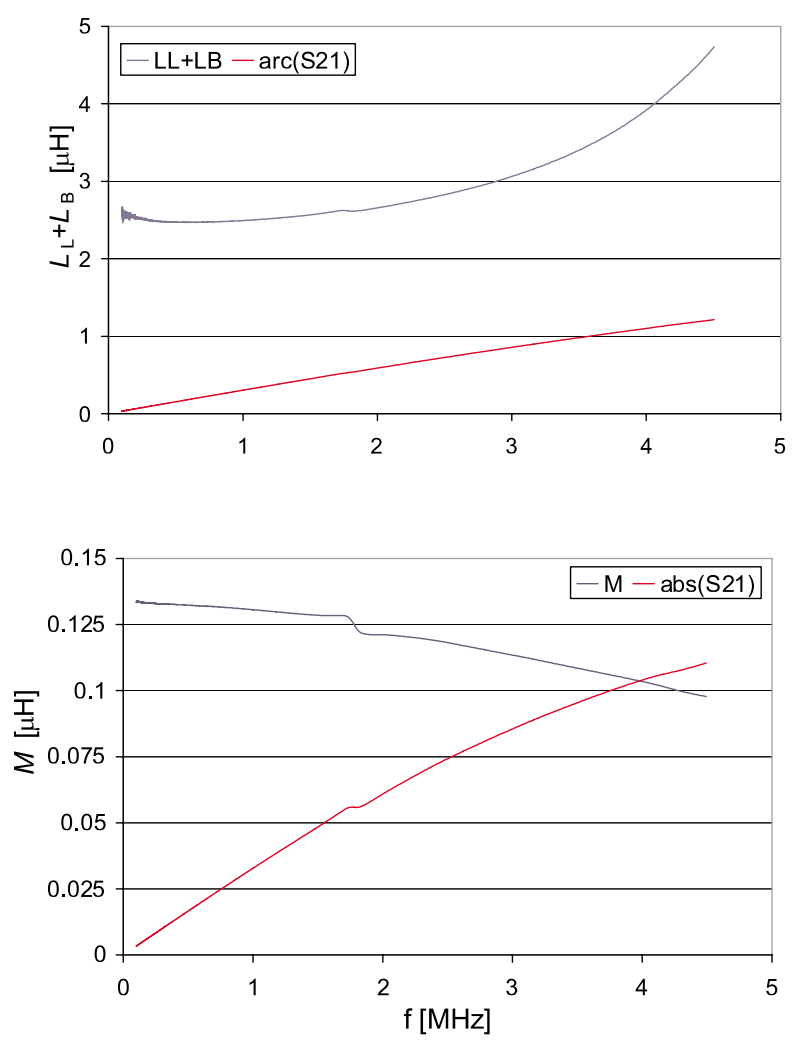

FIG. 9. (Color) Mutual inductance from the busbar to the TWline scattering coefficient.

$M h / \Delta \approx 0.80 \mu H$, a value close to but, as required, below the measured $L_{B}=0.954 \mu \mathrm{H}$. Subtracting the relevant values yields the side-strap inductance of $L_{S}=$ $L_{B}-L_{K} \approx 0.154 \mu \mathrm{H}$. Seen from the primary beam side, the side-strap inductance is part of the external kicker termination and could be taken into account for direct coupling impedance measurements via the ratio $L_{K} / L_{D} \approx 0.85$ as corrective factor. The correction is comparable to instrumental errors and will be neglected in the direct coupling impedance measurements unless explicitly mentioned. As expected, the coupling of twin wire and busbar, when expressed by the coupling coefficient $\kappa \approx 0.134$, is weak.

\section{DIRECT COUPLING IMPEDANCE MEASUREMENT}

The frequency range of interest to SNS covers the frequency range from $\sim 100 \mathrm{MHz}$ down to below $1 \mathrm{MHz}$. Especially at the very-low-frequency end, the signals from the wire measurement become very weak and the results appear extremely noisy. This provided the renewed motivation to investigate the wireless direct impedance measurement as a complementary method. Coupling impedance measurements without wires have been reported in the literature [13] and preliminary results for the SNS extraction kicker were obtained with this method [7,11]. 
In this method the data is collected directly by an input impedance measurement at the busbar terminal. For the data interpretation, the busbar plates are considered as twin wires with an effective spacing of $h$. The signal-tonoise level in the direct method is thus increased by $(h / \Delta)^{2}=36$ over the wire method. The input impedance $Z^{D}$ yields the vertical coupling impedance according to

$$
Z_{\perp} \approx \frac{c}{\omega h^{2}} Z_{D}
$$

The transformer model suggests a complete agreement as to the "coupled" impedance between the direct and the twin-wire measurement by taking the busbar short as reference. Obviously, neither the leakage inductance nor the small image-induced intrinsic impedance appears in the direct impedance result. Furthermore, only measurements in the kick direction are possible and measurements can only be performed on kicker magnets with accessible busbar terminals. As discussed above, access to the coupled impedance part can be restricted by an inductance, such as the side-strap inductance in the SNS magnet, and could be corrected.

The viability of the direct method has been explicitly verified by comparison with the results from two different wire measurements, with and without transformers. In the low-frequency range, the twin-wire line can also be used without transformer or resistive matching to increase the signal strength by a factor of 5 (i.e., 250/50), and to eliminate calibration errors. Transformerless wire measurements uniquely define the use of the instrument impedance $R_{0}$ as the nominal impedance $R_{C}=R_{0}$ rather than the line impedance $Z_{L}$ for the interpretation of the scattering coefficients in Eq. (1). However, there is a systematic error in the scattering coefficient ratio which increases with frequency and is approximated by [4]

$$
\delta\left(\frac{S_{21}^{\mathrm{DUT}}}{S_{21}^{\mathrm{REF}}}\right) \approx j 2 \ell_{L} \frac{\omega}{c} \frac{Z^{\mathrm{DUT}}}{Z_{L}} \frac{Z_{L}^{2}-R_{0}^{2}}{\left(2 R_{0}+Z^{\mathrm{DUT}}\right)^{2}} .
$$

The low-frequency transverse impedance from the direct and twin-wire measurement of the bare kicker magnet without feedthrough but with a $25 \Omega$ termination are compared in Fig. 10. The data from the wire measurement with transformer (TW w X, yellow) become noisy below $\sim 3 \mathrm{MHz}$. The wire measurement without transformer (TW wo X, red) is less noisy and serves as calibration for the direct measurement (DRCT, blue). The peak impedance at $\sim 4 \mathrm{MHz}$ from the direct measurement differs from the wire measurement only by the corrective factor of $\sim 0.85$ discussed above.

The transformerless wire method is limited to low frequencies whereas the direct method can be extended to higher frequencies. The agreement with wire measurements confirms the direct method as a valid alternative to the transverse impedance measurement. It has the highest signal-to-noise ratio and in fact can serve

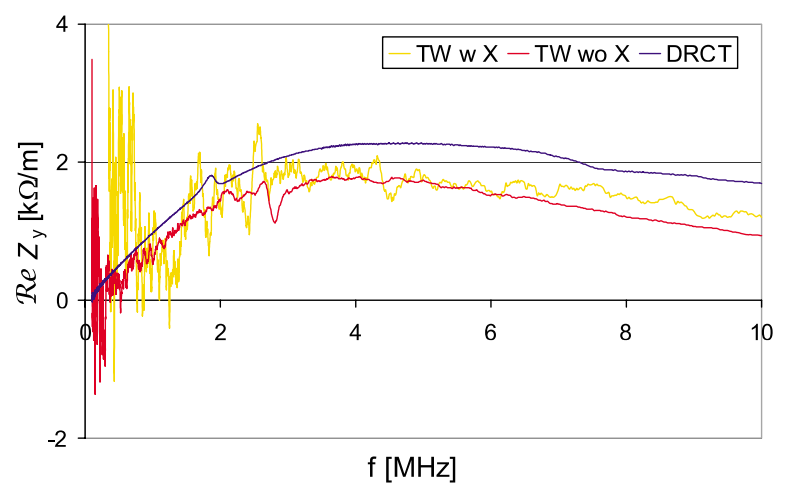

FIG. 10. (Color) Transverse impedance from wire and direct measurements.

for production measurements (even without correction). Notwithstanding its limitations, the direct coupling impedance measurement turns out to be the preferred method below $\sim 10 \mathrm{MHz}$.

\section{DUT IMPEDANCE OF THE PROTOTYPE KICKER MAGNET}

The transverse DUT impedance of the bare SNS kicker magnet, i.e., with the feedthrough and PFN disconnected, was measured using the wire as well as the direct method. The measured transverse impedance, here referred to as total impedance, is composed of the impedance coupled to the termination plus the intrinsic impedance plus the leakage inductance. The separate contributions can be identified by making measurements with appropriate configurations on the shorted, open, and terminated magnet, respectively.

For the wire measurements, the TW line was assembled with the X200-100 or the X200-60 transformers and was matched to $R_{C}=250 \Omega$. In order to work with an acceptable signal-to-noise level on one hand and to remain in the perturbative region on the other hand at all frequencies, the wire spacing was kept at $\Delta=40.6 \mathrm{~mm}$. The validity of a bench measurement, that is the degree to which the measured impedance value corresponds to that seen by the beam, is more difficult to ascertain in the transverse than in the longitudinal case. The validity could be increased by reducing the wire spacing thereby reducing the coupling coefficient and thus the leakage inductance, which is not seen by the beam. In the present setup with $\Delta=40.6 \mathrm{~mm}$, the measured leakage inductance is estimated as $\kappa^{2} L_{L} \approx 0.021 \mu \mathrm{H}$ which is comparable in size to $L_{K}(\Delta / h)^{2} \approx 0.022$ seen by the beam and thus represents a limit on the validity of the wire method as to the reactive impedance component. Note that $\kappa$ impacts only the inductive component and fortuitously leaves the validity of the resistive part unchanged.

Separating the impedance parts involves reference calibration of the scattering coefficient $S_{21}^{\mathrm{REF}}$ versus the 
tube (vT) or against the shorted busbar (vS). The primary measurement is done versus the tube and yields the total impedance, which, however, differs from the total beam impedance by the leakage inductance impedance. Measuring the magnet with the busbar shorted, still versus the tube, yields the intrinsic impedance and the leakage inductance. Subtracting the shorted from the total impedance yields the coupled termination impedance. Alternatively, measuring the magnet versus the shorted busbar also provides the coupled termination impedance and serves as error check.

In the low-frequency range of interest to the SNS performance, $\sim 1$ to $10 \mathrm{MHz}$, the intrinsic impedance is small and the resistive transverse impedance is primarily due to the coupled termination impedance. The measurement versus short leads to the elimination or at least a reduction of errors due to mechanical changes or drift in the network analyzer. It follows that using the shorted busbar as reference gives the more accurate results for the coupled impedance at low frequencies. Note that the result for the resistive impedance component, which is of particular interest for the beam stability, remains unchanged by the choice of the (lossless) reference calibration, although the reactive part is modified.

Further measurements were performed using the direct method, which as discussed above, provides noise free data down to the lowest frequencies. The direct method can only give the coupled impedance and is expected to agree with the measurement versus short, if the small side-strap correction can be ignored.

\section{A. The shorted magnet}

The DUT impedance of the magnet with shorted busbar is shown in Fig. 11. The magnet was wire measured versus the tube reference using the X200 transformers, with the transformers identified as to their $100 \mathrm{MHz}$ (vT $\mathrm{X}-100$, blue) and $60 \mathrm{MHz}$ (vT X-60, red) frequency range. The wire measurements with the two transformers agree up to $\sim 20 \mathrm{MHz}$, but differ at higher frequencies and point to the accuracy limit of wire measurements. Overall, the X-100 transformer seems more credible for measurements covering the entire frequency range and will be preferred.

The wire measurements vT here yield the sum of intrinsic impedance plus the leakage inductance together, but their contributions can be separated as follows. The imaginary part of $Z^{\text {DUT }}$ in Fig. 11 gives the low-frequency inductance to be $\sim 0.02 \mu \mathrm{H}$, a value within measuring accuracy equal to and thus identifiable as the leakage inductance $\kappa^{2} L_{L}=0.021 \mu \mathrm{H}$. By implication, the intrinsic impedance is essentially resistive and can be obtained from the real part of $Z^{\text {DUT }}$ in Fig. 11 to be $L_{I} \approx$ $-j 0.003 \mu \mathrm{H}$. The intrinsic impedance is small and independent of the load, and is generated at higher frequencies by the magnet ferrite losses.
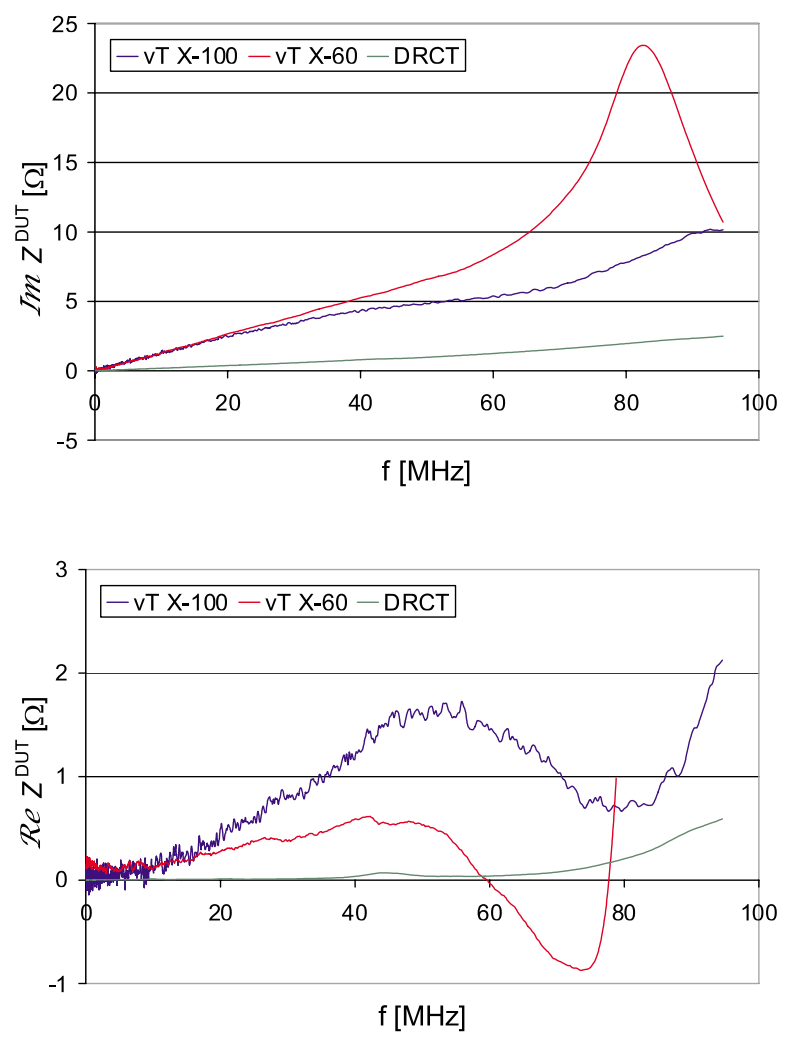

FIG. 11. (Color) Measured DUT impedance of the magnet with shorted busbar.

The wire results are shown in Fig. 11 together with the direct data (DRCT, green), scaled for comparison by the factor $(\Delta / h)^{2}=1 / 36$. The direct measurement represents the inductance of the magnet $L_{D}$ with the shorting strap in parallel, and thus is generated primarily by the strap inductance of $\sim 0.003 \mu \mathrm{H}$. Since imperfect, the short allows at higher frequencies small busbar currents and some ferrite losses, but the deviations from short are for all practical purposes negligible.

\section{B. The open magnet}

The DUT impedance of the magnet with open busbar is shown in Fig. 12. The impedance measured versus tube (vT, blue) is shown together with the measured impedance versus short (vS, red). The measurement vT represents the sum of coupled plus intrinsic plus leakage impedance. Consistency of the measurements is demonstrated by adding the impedance versus short of the open magnet (vS, red) to that of the shorted magnet vT (SHRTvT, X-100 in Fig. 11) and by comparing the sum (vS + SHRTvT, yellow) with the total impedance vT. Full agreement is found, even though the three measurements were performed at different times and thus provide an indication of the measuring accuracy.

The direct measurement (DRCT, green, also shown in Fig. 8) can only give the coupled impedance and thus it must be compared with the equivalent impedance versus 

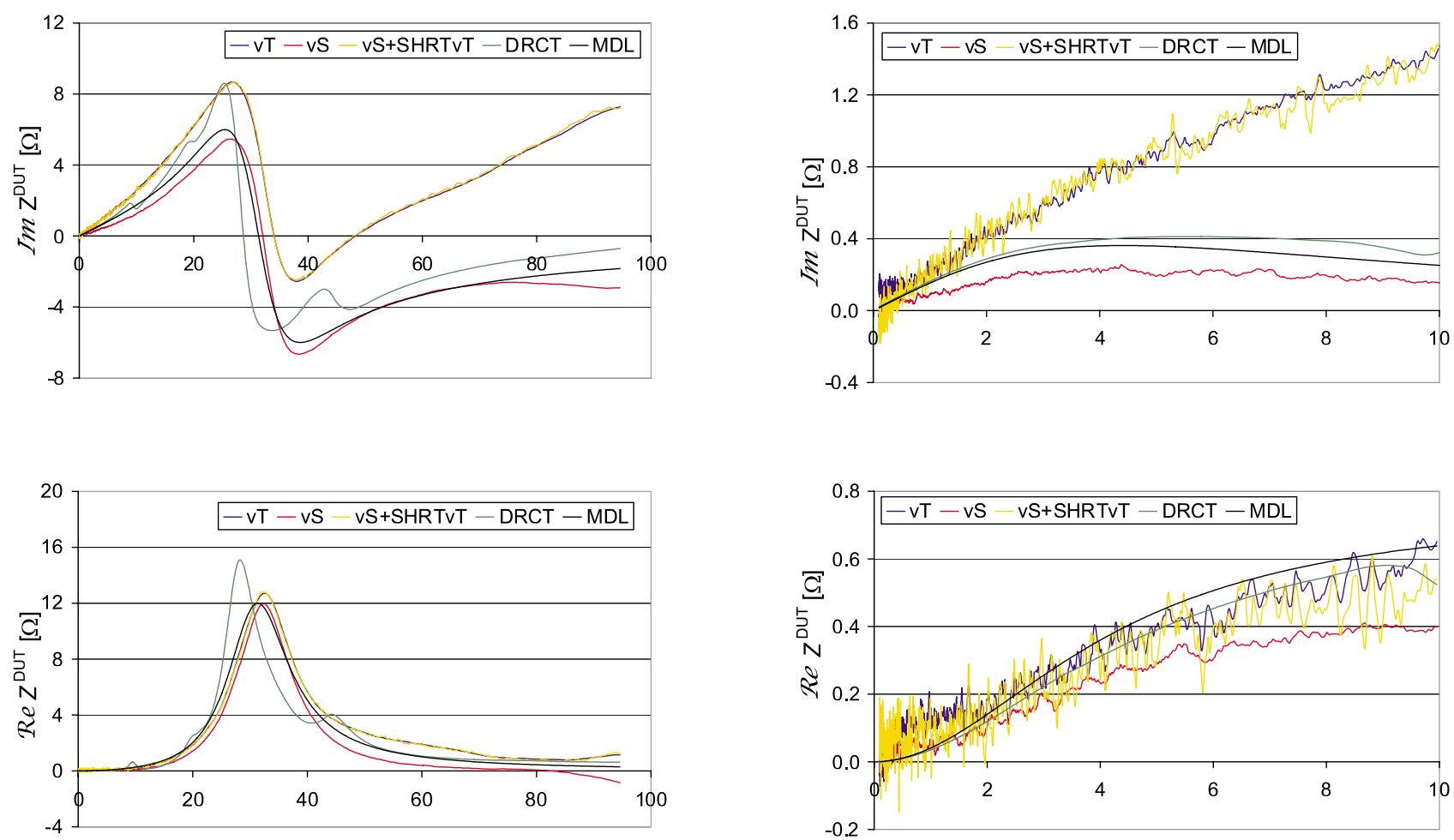

$\mathrm{f}[\mathrm{MHz}]$

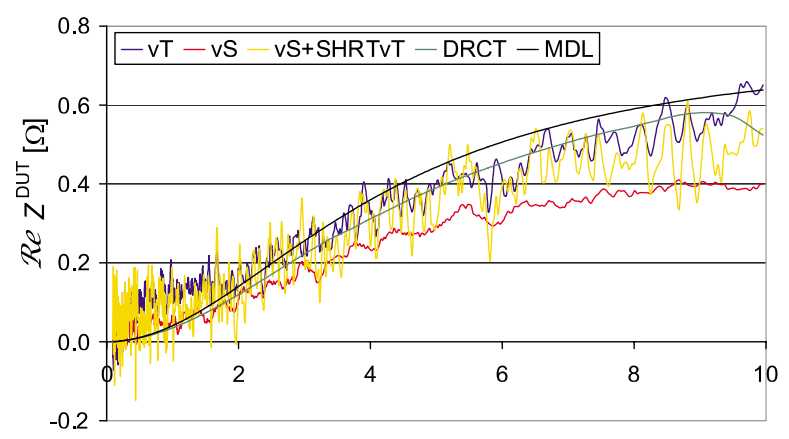

$\mathrm{f}[\mathrm{MHz}]$

FIG. 12. (Color) Measured DUT impedance of the open kicker magnet.

the shorted busbar (vS, red). The coupled impedance alone can be represented numerically in the transformer model by a resonant circuit (MDL, black) with the circuit parameters of $L_{B}=0.954 \mu \mathrm{H}, C_{B}=27 \mathrm{pF}$, and a parallel resistor. The value of the resistor is obtained at the busbar from the direct impedance peak with the sidestrap correction applied, $Z^{D}=620 \times 0.85=530 \Omega$ and is in good agreement with the impedance vS by scaling of its peak value $R=(h / \Delta)^{2} 12.7 \Omega \approx 460 \Omega$. The resonance shift from $\sim 32$ to $\sim 28 \mathrm{MHz}$ is due to the clip lead capacity in the direct measurement.

Overall, the various measurement methods, even uncorrected, give results for the total resistive component of the open magnet which are sufficient for quantitative survey measurements. The difference in the reactive part of the total impedance from the wire vT on one hand and the wire vS or direct method on the other is attributable primarily to the leakage inductance inherent to the twin-wire measurement method.

\section{Magnet with $25 \Omega$ termination}

The measured DUT impedances for the prototype kicker magnet, terminated at the busbar with $25 \Omega$, are shown in Fig. 13 for the low frequency and in Fig. 14 for the full frequency range. The nomenclature and colors are here retained from Fig. 12. The presence of a termination changes the coupled but not the intrinsic image imped-

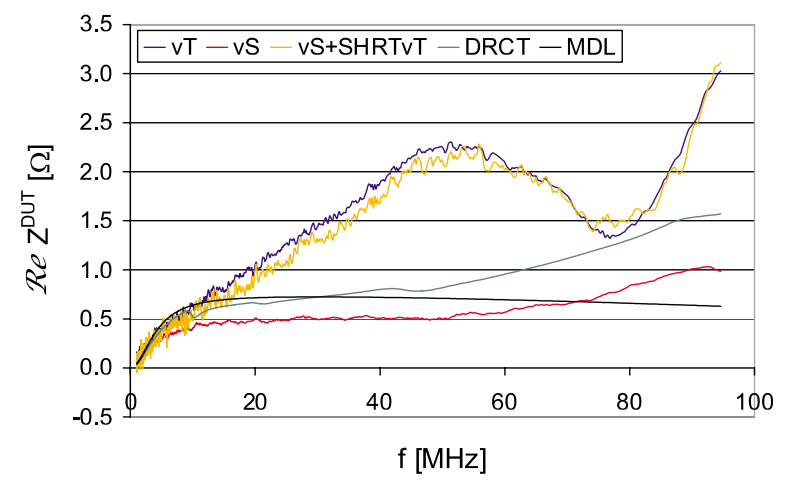

FIG. 14. (Color) Measured DUT impedance of magnet with $25 \Omega$ termination. 
ance nor the leakage inductance. The numerical level of the impedance is here significantly lowered, which makes the data and their interpretation more sensitive to small errors.

The results clearly indicated two frequency regions as to the possible measuring methods beyond the measurement vT. At low frequencies, below $\sim 10 \mathrm{MHz}$, the resistive impedance component is determined by the coupled impedance, dominated by the $25 \Omega$ termination, and can also be measured versus short or with the direct method. At higher frequencies the intrinsic impedance due to image currents becomes comparable to the load generated impedance and measurements vT (blue) or the sum (yellow) of vS plus SHRTvT are required.

In summary, interpreting the collective set of magnet measurements confirms wire measurements versus a beam tube calibration as the primary method to find the transverse coupling impedance at most frequencies of interest here. However, the signal-to-noise ratio decreases at lower frequencies to a degree that the data below a few $\mathrm{MHz}$ becomes marginal. The frequency range of valid data can be extended for the instability driving resistive part of the impedance by making wire measurements versus the shorted busbar as reference. Alternatively, the direct impedance measurement at the busbar, even without the correction for the strap inductance, provides low noise and reliable results from $\sim 10 \mathrm{MHz}$ down to a few $100 \mathrm{kHz}$. Obviously, the use of several complementary methods will increase the credibility of the results presented.

\section{PFN MAGNET TERMINATION}

The kicker magnet is located in a vacuum vessel with a feedthrough and is electrically connected to the PFN by means of two $\sim 200 \mathrm{~m}$ long $50 \Omega$ cables. The cables are in parallel to match to the nominal $25 \Omega$ PFN output impedance. Wire measurements of the kicker system, that is the magnet with PFN connected, can only be done with the vessel open. In air, the magnet busbar terminals as well as the feedthrough terminals are accessible, allowing input impedance measurements at various points of the system components, separately or connected.

Deviations, resistive or reactive, from the ideal $25 \Omega$ PFN impedance lead to frequency dependent impedance oscillations at the busbar terminals and consequently of the kicker coupling impedance. The impedance seen directly at the busbar is shown in Fig. 15 for the prototype magnet terminated either with a local $25 \Omega$ resistor (green), with the cables terminated by a perfect $25 \Omega$ (blue), or with the first-article production PFN (P 1, red) as delivered.

The results pointed to a significant increase of the coupling impedance with the production PFN compared to previous results obtained with the prototype PFN. At first, the presence of a saturating inductor in the PFN was suspected, but shorting it greatly increased the mismatch.
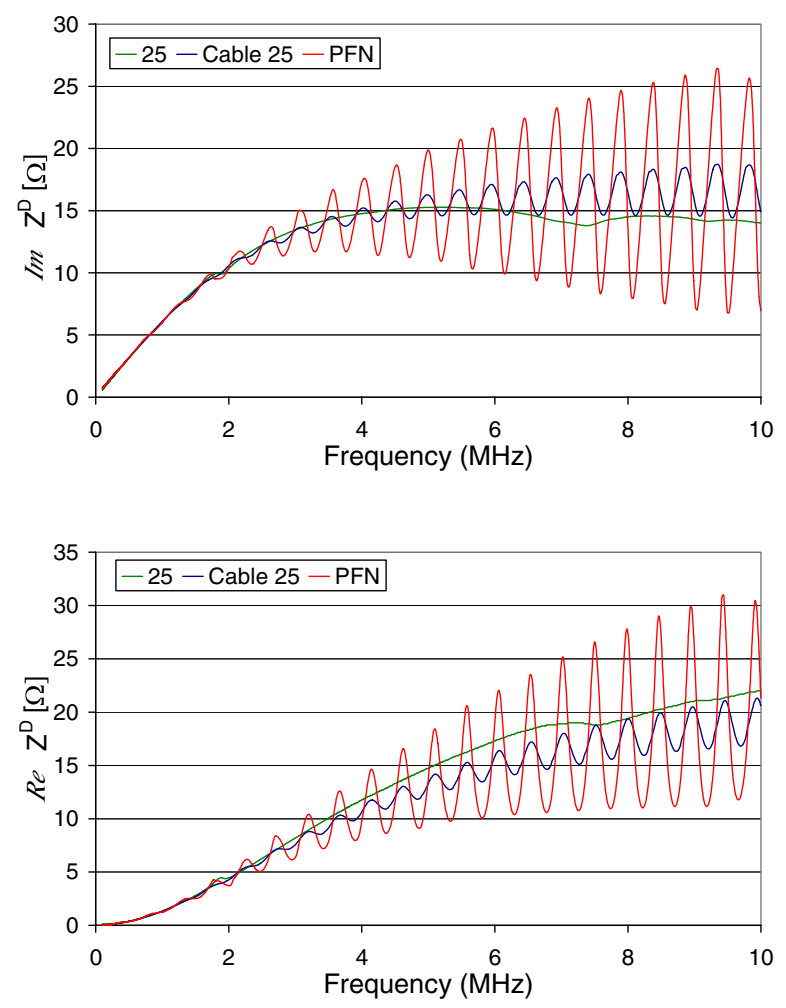

FIG. 15. (Color) Direct impedance with $25 \Omega$, cable with $25 \Omega$, or cable with PFN.

Correction of grounding problems in the production PFN reduced but did not eliminate the mismatch.

Input impedance measurements were then made at the PFN terminals to identify the source of the mismatch and to guide in its correction. The input impedance of the production PFN as delivered ( $\mathrm{P}$ 1, black) is shown in Fig. 16. Comparing the production with the prototype PFN (not shown) pointed to an increased inductive component in the production unit as the culprit. Finally, the inductance of the large PFN load resistor was identified as the inductive source, and the inductance reduction of the PFN by adding a coaxial screen over the large load resistor was successfully demonstrated (w Scrn, green). The impedance of the freestanding screened load resistor ( $\mathrm{R}$ load, yellow) is also shown in Fig. 16, and the production units are now delivered with a screened load resistor.

The improvement from the coaxial screen was demonstrated by measuring the transverse coupling impedance of the prototype magnet with the first-article PFN in its various stages. The dominant instability driving resistive coupling impedance in the vicinity of the low-frequency peak was obtained from a direct measurement with the scaling of $Z^{\text {DUT }}=Z^{D}(\Delta / h)^{2}$ applied. The transverse coupling impedance is shown in Fig. 17 for the PFN without screen (no Scrn, yellow), and as modified with the coaxial screen (Scrn, blue). The peak value is effectively reduced (by about 25\%) when compared to the PFN without screen. If necessary, the PFN inductance can be further 

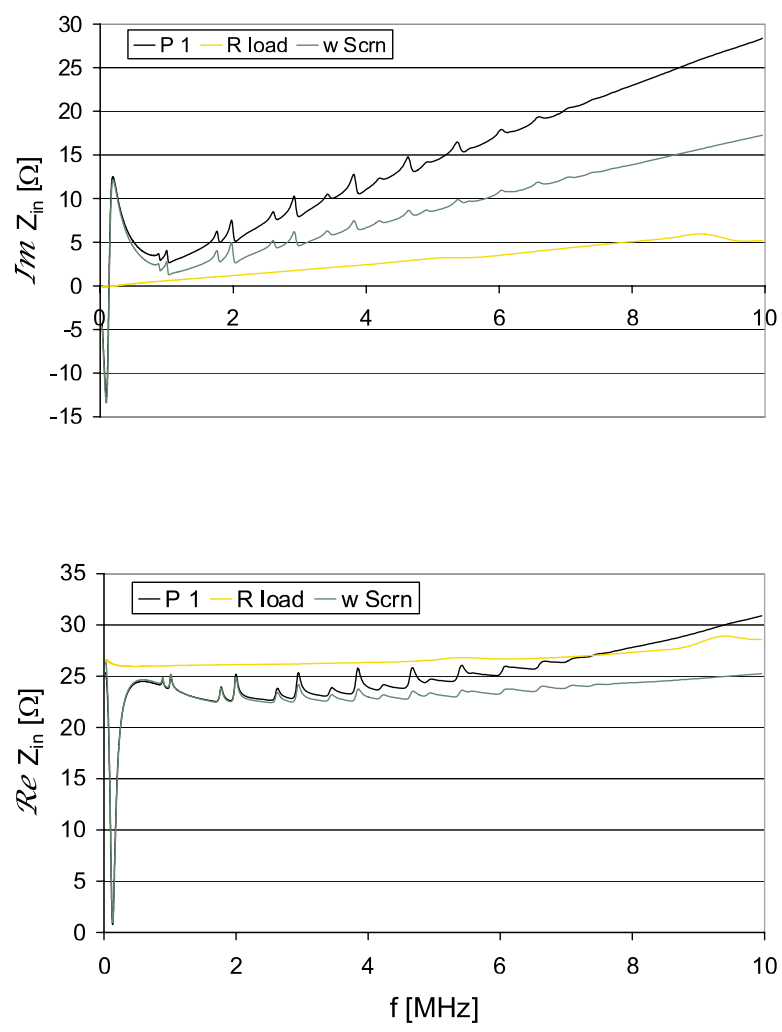

FIG. 16. (Color) Input impedance of the PFN.

reduced by adding a resistor and capacitor (RC) shunt with a series $R=25 \Omega$ and $C=100 \mathrm{pF}$ across the PFN terminals (Scrn $+\mathrm{R} \& \mathrm{C}$, green). The gain from a $\mathrm{RC}$ shunt alone $(\mathrm{R} \& \mathrm{C}$, red) almost equals that from the screen, but is inferior to the solution from the combination of the screen plus a RC shunt ( $\mathrm{Scrn}+\mathrm{RC}$, green). It is considered an option in reserve to be added if required by operation.

The wire impedance at frequencies beyond $\sim 10 \mathrm{MHz}$ gains less from these modifications as seen in Fig. 18. The input impedance with screen (Scrn vT, blue) is smaller, but not significantly below the unscreened (no Scrn vT, yellow) data. The cables have high-voltage connectors at

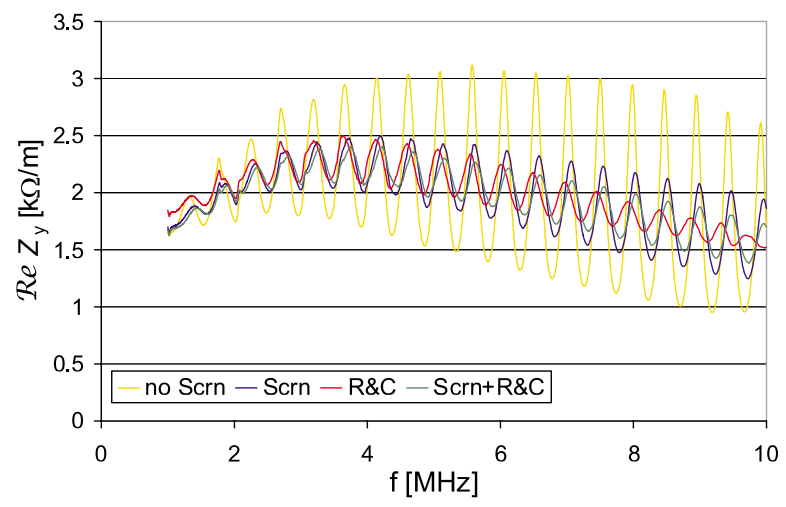

FIG. 17. (Color) Transverse impedance with modified production PFN.
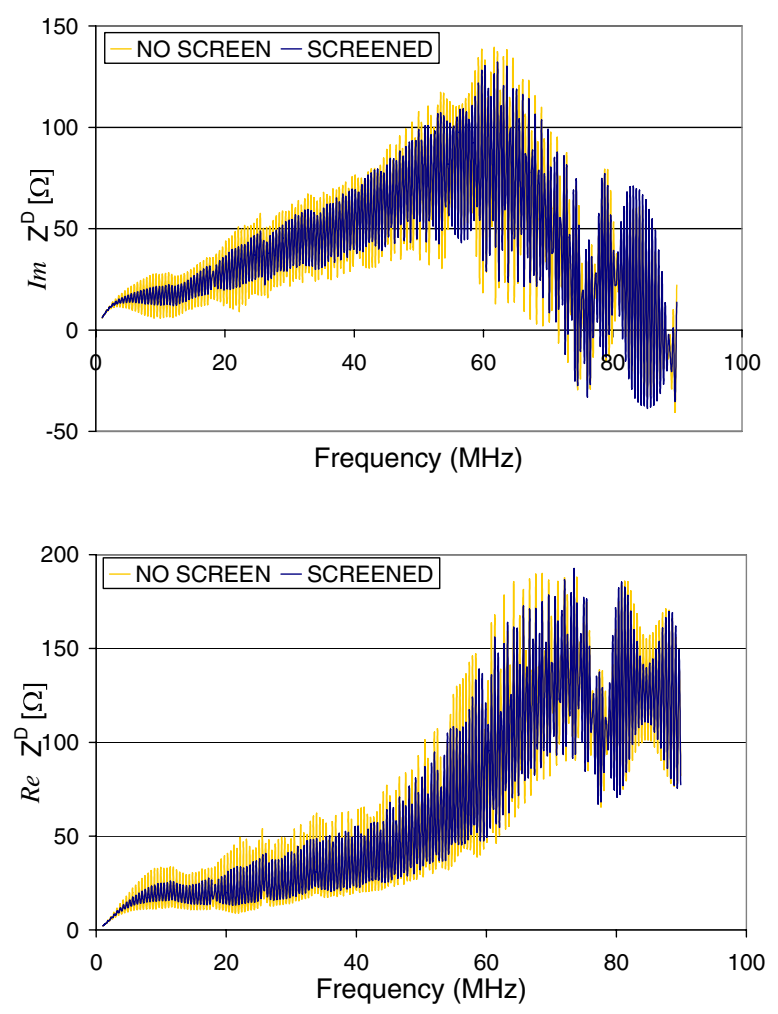

FIG. 18. (Color) Impedance seen at the busbar of the magnet with PFN.

either end and the feedthrough is a nonmatched magnet vessel penetration, so that an irreducible impedance increase over perfectly matched cables must be accepted at higher frequencies.

\section{TRANSVERSE COUPLING IMPEDANCE OF THE PROTOTYPE KICKER SYSTEM}

The transverse impedance is obtained from the device under test impedance as discussed above. The transverse impedance differs in the horizontal and the vertical direction. The vertical impedance $Z_{y}$ is in the kick direction and depends on the kicker magnet termination. The horizontal impedance $Z_{x}$ has a character similar to the "intrinsic" vertical impedance, and is mostly inductive with a ferrite-generated resistive component. In this section, results for the extraction kicker system consisting of the prototype magnet and the first-article PFN after all its modifications are presented and discussed.

\section{A. Vertical coupling impedance}

The vertical transverse impedance of the kicker system covering the full frequency range of interest, i.e., from $\sim 1$ to $100 \mathrm{MHz}$, was obtained from the wire method versus tube, versus short, as well as the direct impedance measured at the busbar. The wire measurements were averaged over three sweeps, and unless noted otherwise are without smoothing. The results are presented in 
Fig. 19 for the low-frequency range and in Fig. 20 for the full frequency range, obtained with the X200-60 and X200-100 transformers, respectively.

The signal-to noise-ratio below $\sim 10 \mathrm{MHz}$ practically prevents the acquisition of valid data for the resistive component by the wire measurement (vT, yellow), in particular, for the resistive peak value at $\sim 4 \mathrm{MHz}$. Here the application of the direct impedance (DRCT, black) is indicated and yields reliable resistive values. The reactive component can be obtained with the wire measurement (vT, yellow). Alternatively, it is found as the sum (DRCT + SHRTvT, blue) from the direct (DRCT, black) plus the value from wire measurement of the shorted magnet (SHRTvT, red). The latter is given by the leakage inductance, an effect due to the bench measurement, and not seen by the beam.

The results for the full frequency range in Fig. 20 are obtained with the wire method, both without smoothing (vT $0 \mathrm{sm}$, blue) and with $0.1 \%$ smoothing (vT $0.1 \mathrm{sm}$, yellow). Also shown is the intrinsic impedance for the shorted busbar (SHRT, red). Adequate overlap of the results in Fig. 20 with those in the low-frequency region (Fig. 19) is found. The resistive impedance is generated by the termination plus the image-current induced part. The imaginary impedance is primarily due to the leakage inductance since the busbar plate capacitance effectively shorts the termination at higher frequencies.
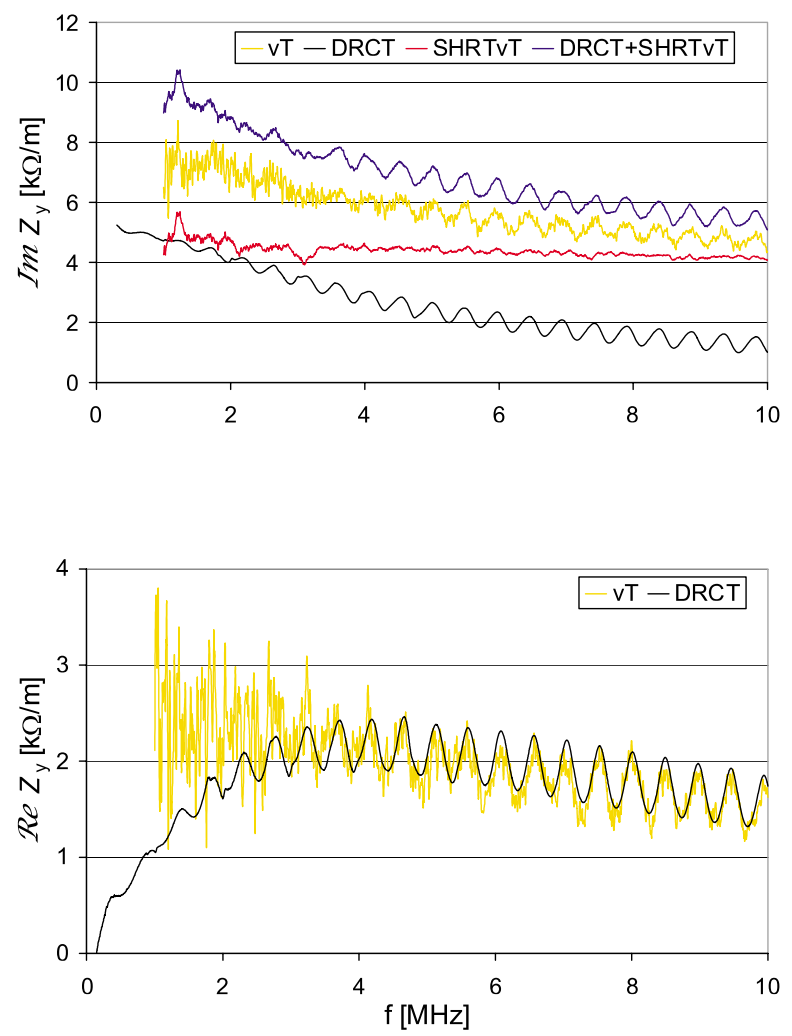

FIG. 19. (Color) Vertical impedance of kicker system at low frequencies.
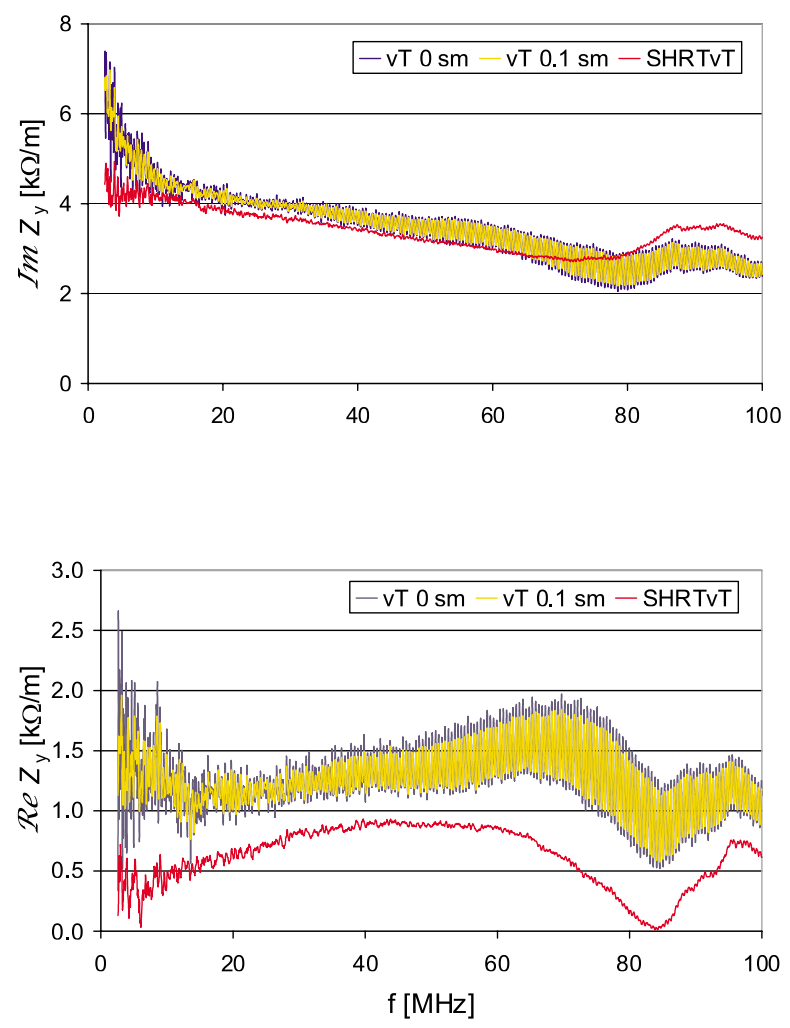

FIG. 20. (Color) Vertical Impedance of the kicker system.

\section{B. Horizontal Impedance}

The horizontal impedance is independent of the busbar termination and image current induced and is here primarily a reactive value with small ferrite losses. The results for the full frequency range are obtained with the wire method using the X200-100 transformer and are shown in Fig. 21.

\section{SYSTEM IMPEDANCE WITH THE SMALL- SIZE MAGNET K11}

The entire SNS extraction kicker system consists of 14 individual magnets. The kicker construction of these magnets is similar, although with different geometrical dimensions. The vertical height of the production mag-

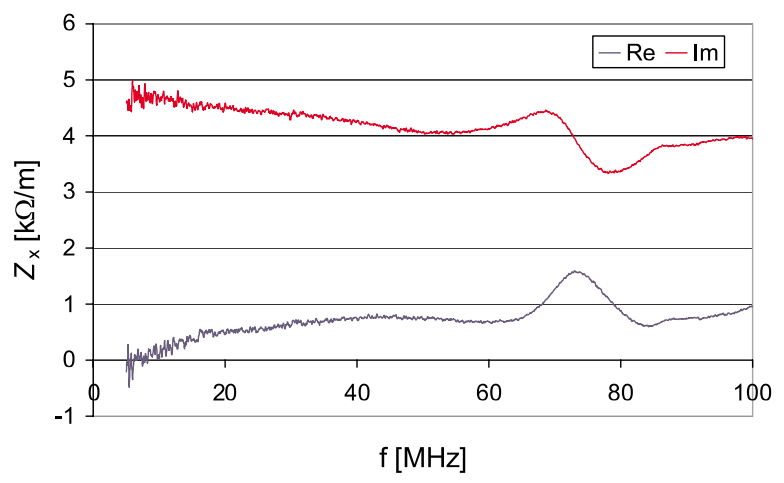

FIG. 21. (Color) Horizontal impedance of the kicker system. 
nets ranges from 24.3 to $16.6 \mathrm{~cm}$ measured between the busbar plates, or 24.8 to $17.1 \mathrm{~cm}$ between ferrites. The horizontal widths and lengths range from 12.0 to $21.1 \mathrm{~cm}$ and 39 to $51 \mathrm{~cm}$, respectively. The recent availability of the smallest kicker K11 provided the opportunity for additional measurements which can be used for a review of impedance scaling with geometry and improved estimates of the entire system. The geometrical dimensions of the $\mathrm{K} 11$ magnet are $h=17.1 \mathrm{~cm}, w=12.0 \mathrm{~cm}$, and $\ell=$ $35 \mathrm{~cm}$ leading to an inductance of $L \approx \mu_{0} h(\ell+w) / w=$ $0.842 \mu \mathrm{H}$. The measured input impedance of the open K11 magnet (blue) is shown in Fig. 22 with the inductance at $f=1.5915 \mathrm{MHz}$ as $L=0.871 \mu \mathrm{H}$. Also shown is the input impedance with a $25 \Omega$ termination (red).

The low-frequency vertical impedance of the K11 magnet with the PFN as termination was measured both by the wire method versus tube (vT $0 \mathrm{sm}$, blue and vT $0.1 \%$ sm, yellow) and the direct method (DRCT, black), with the low-frequency results shown in Fig. 23. At frequencies at and above the $\sim 4.5 \mathrm{MHz}$ peak, the two methods agree rather well for the resistive component. One finds at the resistive peak an impedance of $\sim 4 \mathrm{k} \Omega / \mathrm{m}$, which is larger than the $\sim 2.4 \mathrm{k} \Omega / \mathrm{m}$ in the prototype magnet by a factor of $\sim 1.63$, but less than the expected vertically scaled factor $(24.3 / 16.6)^{2} \approx 2.1$.

The reactive component is intrinsically less noisy and the good results are obtained with the wire method. The
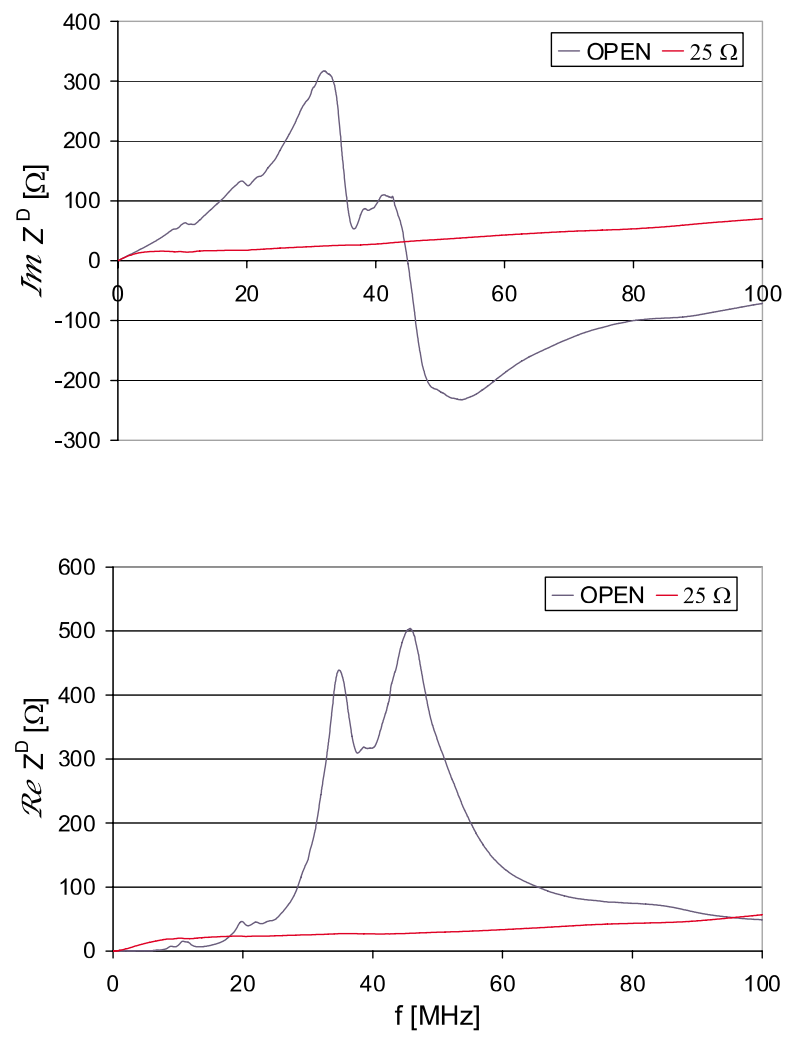

FIG. 22. (Color) Input impedance of $\mathrm{K} 1$ at busbar.
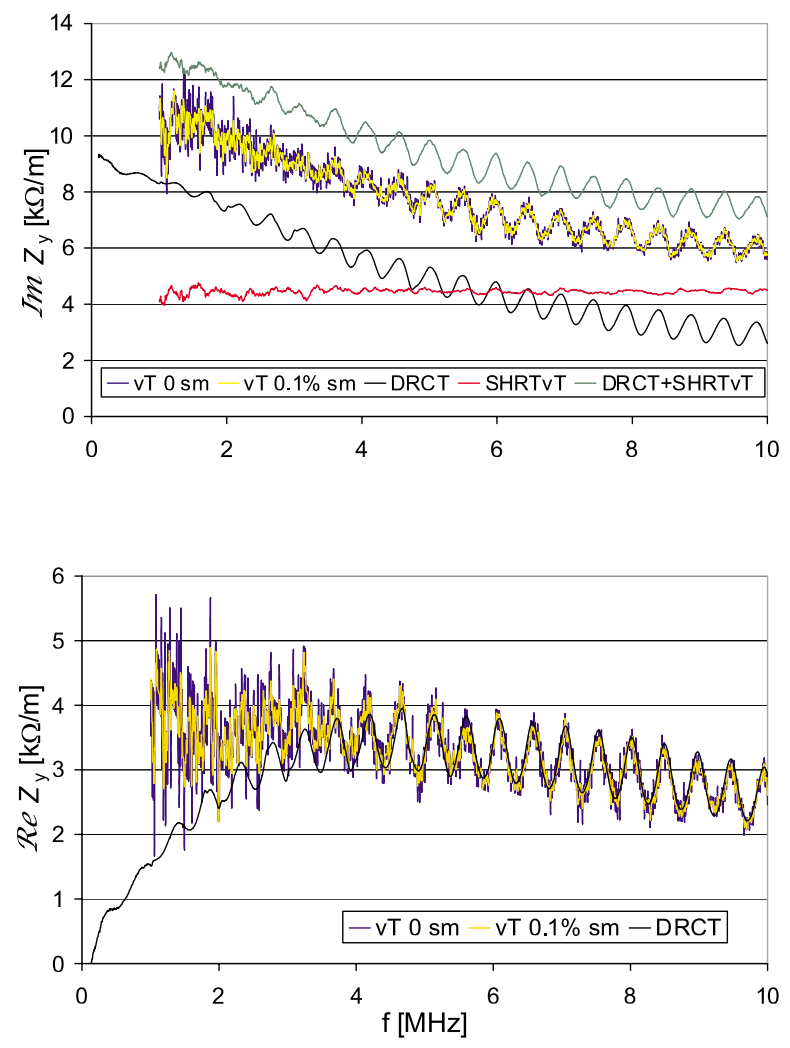

FIG. 23. (Color) Vertical impedance of kicker system with magnet K11.

inductive impedance results for the shorted magnet (SHRTvT, red) are also shown. Adding the inductive component from the direct method to the wire value from the shorted magnet leads to a reasonable agreement $($ DRCT + SHRTvT, green) with the wire method versus tube.

The high frequency measurements of the K11 magnet system were done only with the wire method and without smoothing to retain the oscillations. The results are shown in Fig. 24 for the PFN terminated (vT $0 \mathrm{sm}$, blue) as well as for the shorted K11 magnet (SHRTvT, red). The secondary peak resistive value of $\sim 4.3 \mathrm{k} \Omega / \mathrm{m}$ is here shifted to a higher frequency, $\sim 90 \mathrm{MHz}$. The real part is clearly dominated by the termination, whereas the inductive part is determined by the leakage inductance and possibly by a small image inductance.

The horizontal impedance was measured by the wire method and is shown in Fig. 25. The impedance of both, real and imaginary, is independent of the termination and generated by the image currents, and thus primarily inductive.

\section{IMPEDANCE ESTIMATE FOR THE TOTAL EXTRACTION KICKER SYSTEM}

The availability of the production PFN and of the smallest kicker K11 provided the opportunity for a re- 

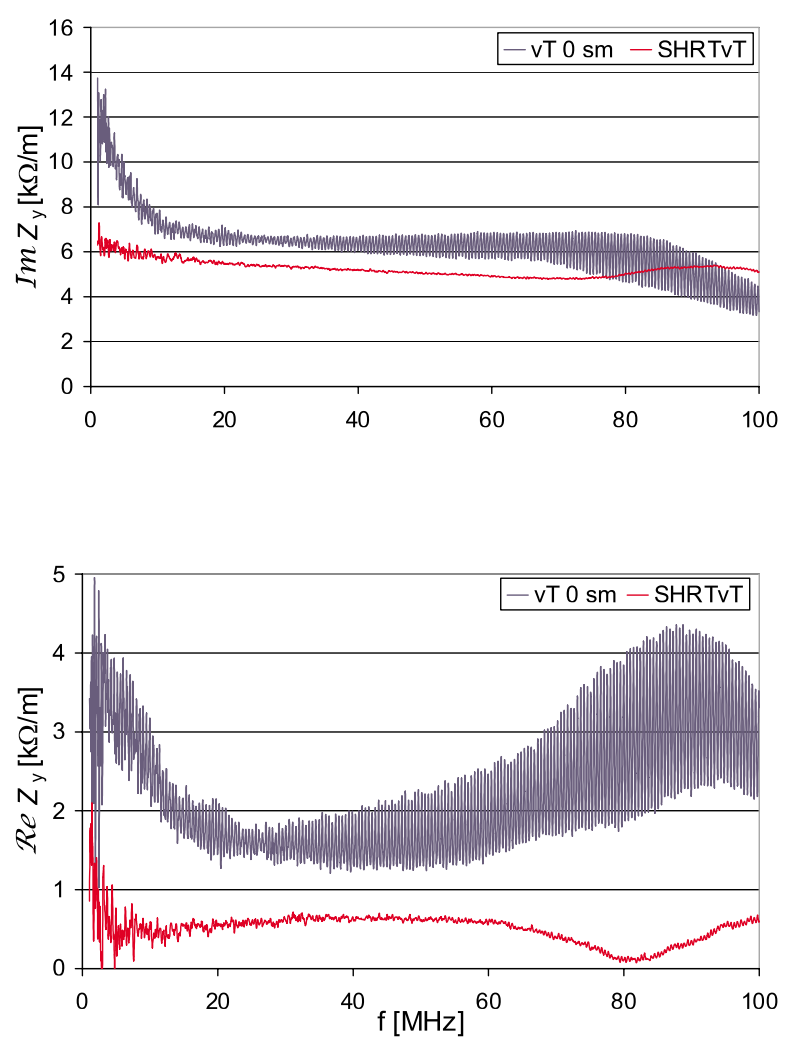

FIG. 24. (Color) Vertical impedance of kicker system with magnet K11.

vised impedance estimate of the total SNS extraction kicker system. The original impedance estimate for the total system was based on the prototype magnet, terminated with a pure resistive $25 \Omega$ termination, and the application of an analytical model [14] for the coupled impedance. The model includes the magnet inductance, busbar plate capacity, and external termination, but its salient feature is the quadratic scaling with the vertical aperture of the kicker magnet. In order to test scaling, and ignoring the small intrinsic impedance, the ratio of the vertical system impedance with K11 divided by that with the prototype is shown in Fig. 26.

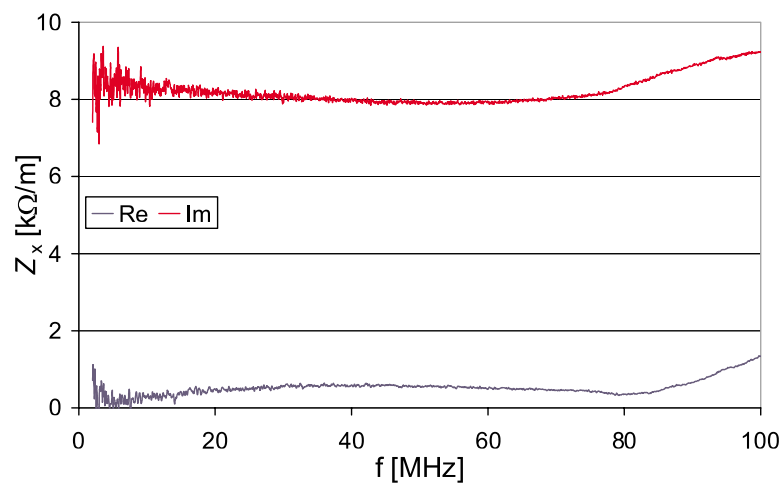

FIG. 25. (Color) Horizontal impedance of K1 kicker magnet.

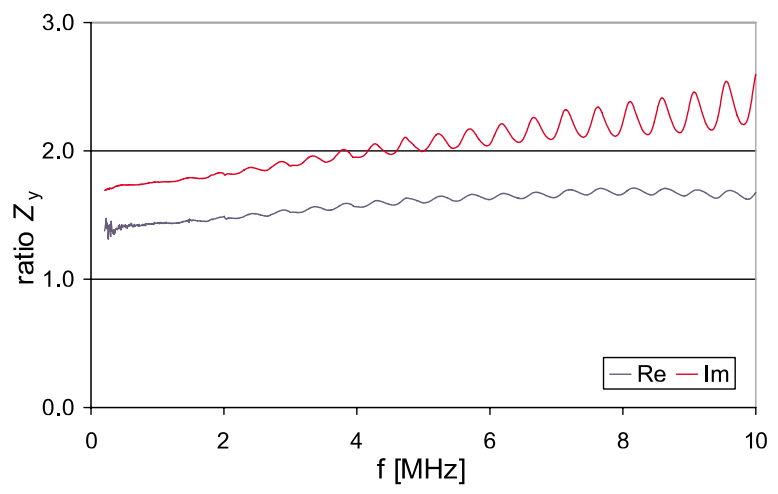

FIG. 26. (Color) Ratio of system impedance with K11 and prototype magnet.

Although not perfect, a quadratic scaling with the gap height and linear with the length can be used for a simplified procedure using only the system impedance of the prototype $Z_{y}(24.3)$ and of the $\mathrm{K} 11 Z_{y}(16.6)$ magnets. The number of magnets with equal height/length is one $(16.6 / 40 \mathrm{~cm})$, two $(19.5 / 51 \mathrm{~cm})$, two $(20 / 40 \mathrm{~cm})$, two $(23.3 / 51)$, four $(23.3 / 43 \mathrm{~cm})$, and three $(24.3 / 39 \mathrm{~cm})$. Summing the scaled contribution from each magnet leads to the expression for the total SNS extraction system impedance

$$
Z_{y} \approx \frac{1}{2}\left[19.93 Z_{y}(24.3)+9.07 Z_{y}(16.6)\right]
$$

leading to the results in Fig. 27. One finds at $\sim 4.5 \mathrm{MHz}$ a resistive impedance peak of $\sim 40 \mathrm{k} \Omega / \mathrm{m}$ which is to be compared with the original estimate of $33.2 \mathrm{k} \Omega / \mathrm{m}$. A secondary peak at the frequency of $\sim 75 \mathrm{MHz}$ has an estimated resistive component of $34 \mathrm{k} \Omega / \mathrm{m}$, significantly higher than the original $12 \mathrm{k} \Omega / \mathrm{m}$, and is attributed to the intrinsic impedance.

As pointed out above, the bench measurements provide valid estimates for the resistive coupling impedance seen by the beam whereas the inductive impedance estimates require a systematic correction for the leakage inductance contribution.

Although the impedance of a single kicker subsystem shows the sharp resonances, one can expect the collective total impedance to be somewhat smeared out. The summation of individual subsystem contributions assumes equal cable lengths, while differences will lower the peaks in the upper frequency region.

\section{ACKNOWLEDGMENTS}

This paper has greatly benefited from the results of previous work performed together with D. Davino. The measurements presented here were done with the help of K. Hartmann and D. Warburton. The author would also like to thank C-I. Pai for mechanical engineering help and the members of the Collider-Accelerator Pulsed 

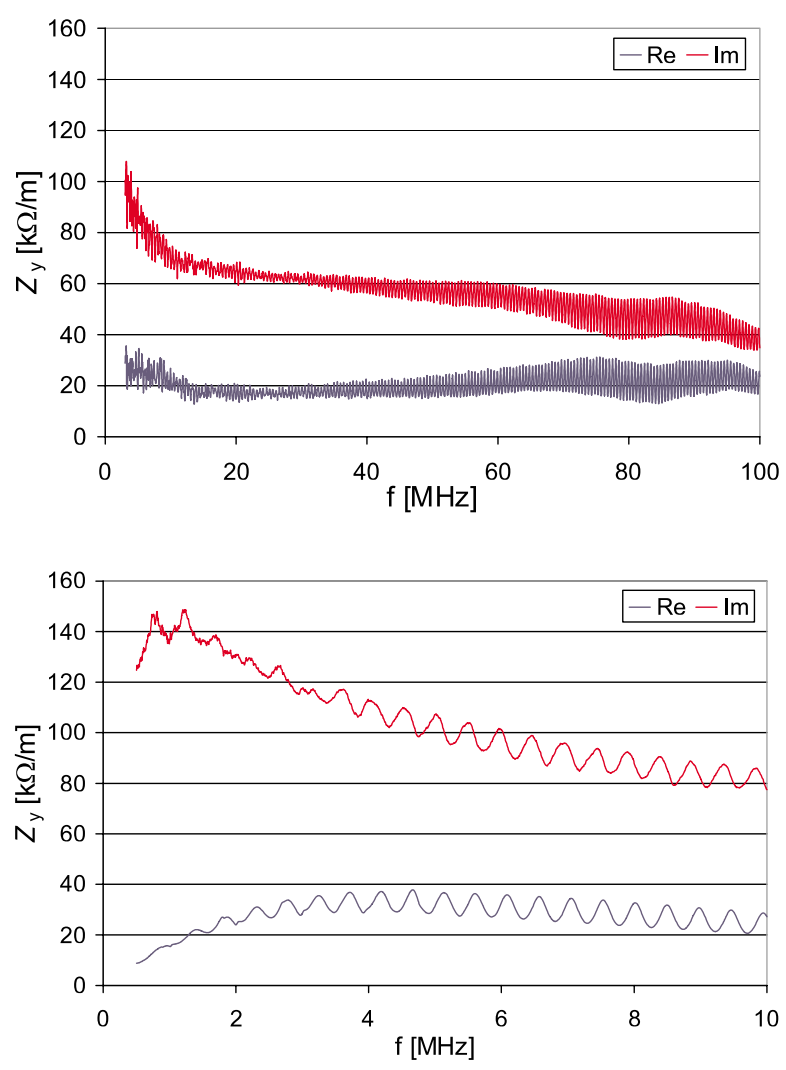

FIG. 27. (Color) Estimated coupling impedance of the SNS extraction kicker system.

Power Group, J-L. Mi, J. Sandberg, and W. Zhang for general support. This work was supported by the SNS project. SNS is managed by UT-Battelle, LLC, under Contract No. DE-AC05-00OR22725 for the U.S. Department of Energy. SNS is a partnership of six national laboratories: Argonne, Brookhaven, Jefferson, Lawrence Berkeley, Los Alamos, and Oak Ridge.

\section{APPENDIX}

The fabrication of magnets, vessels, and PFNs progressed during the review of this paper making the measurement of three additional production magnets, K25, $\mathrm{K} 26$, K27, possible, and leading to additional data important to the project. The magnets have the same geometry, $h=24.3 \mathrm{~cm}, w=15.1 \mathrm{~cm}$, and $\ell=39 \mathrm{~cm}$, but the K26 magnet has a TiN coating for electron-cloud suppression. In this magnet, a $0.1 \mu \mathrm{m}$ thick TiN coating is applied to reduce secondary electron emission from ferrite blocks in order to suppress electron multipacting. The coating is applied in the form of longitudinal stripes, spaced $1.5 \mathrm{~cm}$ apart, and electrically isolated from each other and the busbar.

Establishing the impact of the TiN coating on the coupling impedance represented the primary objective of these measurements. The TiN coated SNS extraction kicker magnet K26 together with two uncoated mag-
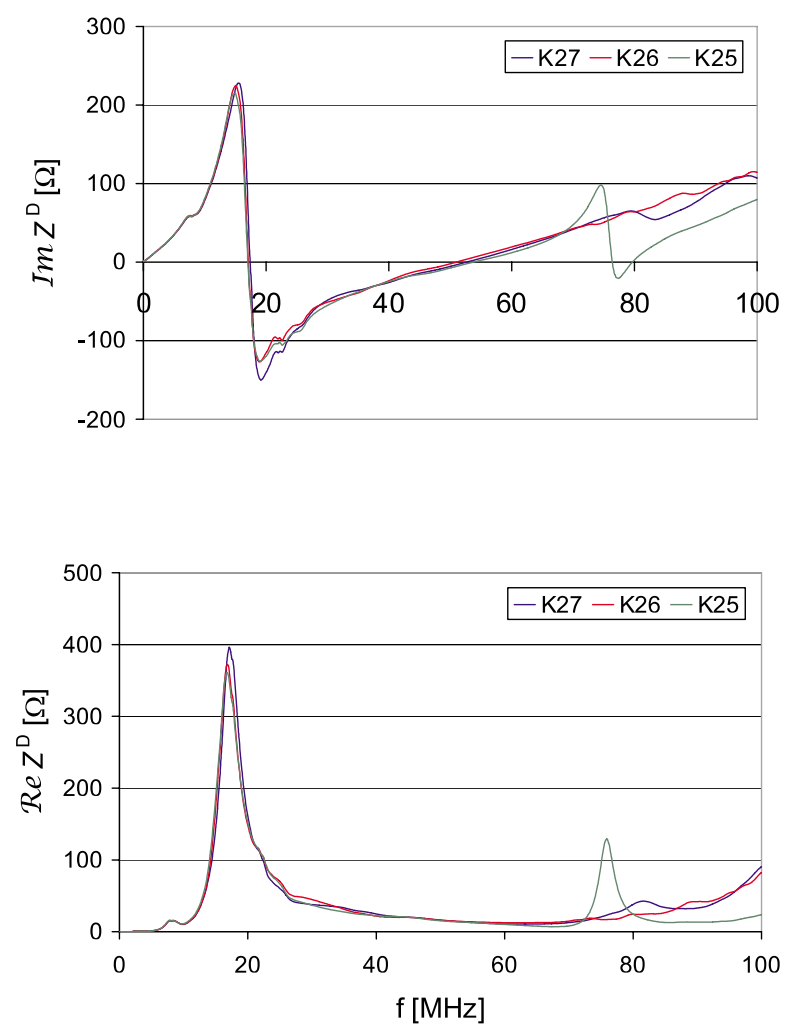

FIG. 28. (Color) Input impedance of production magnets, K26 with TiN coating.

nets were assembled in the downstream vacuum vessel. The inaccessibility of the magnets in the $3.79 \mathrm{~m}$ long vessel made wire measurements practically impossible and singularly pointed to direct impedance measurements. The input impedance of the magnets with feedthrough but disconnected from the PFN is shown in Fig. 28, demonstrating the absence of a coating-induced impedance change.

The geometry and inductance of these magnets is comparable to the prototype, thus projecting the same vertical impedance peak at $\sim 4.5 \mathrm{MHz}$ of $\sim 2.5 \mathrm{k} \Omega / \mathrm{m}$ in Fig. 29.

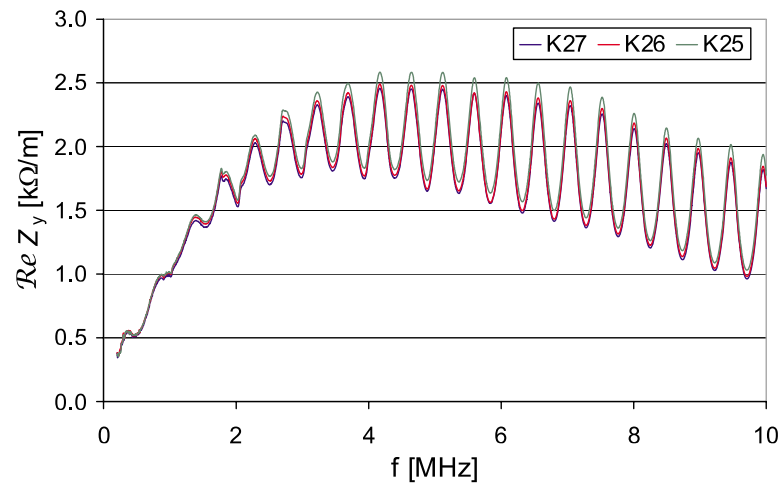

FIG. 29. (Color) Vertical impedance of production kicker systems K25, K26, K27. 
[1] F. Caspers, in Handbook of Accelerator Physics and Engineering, edited by A.W. Chao and M. Tigner (World Scientific, Singapore, 1998), Chap. 7.5.1, p. 570.

[2] G. Nassibian and F. Sacherer, Nucl. Instrum. Methods 159, 21 (1979).

[3] L. S. Walling, D. E. McMurray, D.V. Neuffer, and H. A. Thiessen, Nucl. Instrum. Methods Phys. Res., Sect. A 281, 433 (1987).

[4] H. Hahn, Phys. Rev. ST Accel. Beams 7, 012001 (2004).

[5] J. Wei, in Proceedings of the 2002 European Particle Accelerator Conference, Paris, 2002 (EPS-IGA and CERN, Geneva, 2002), p. 1067.

[6] J. G. Wang and S. Y. Zhang, Nucl. Instrum. Methods Phys. Res., Sect. A 459, 381 (2001).

[7] D. Davino and H. Hahn, Phys. Rev. ST Accel. Beams 6, 012001 (2003).
[8] D. Davino, H. Hahn, and Y. Y. Lee, in Proceedings of the 2002 European Particle Accelerator Conference, Paris, 2002 (Ref. [5]), p. 1467.

[9] H. Hahn and D. Davino, in Proceedings of the 2002 European Particle Accelerator Conference, Paris, 2002 (Ref. [5]), p. 1502.

[10] A. Mostacci, F. Caspers, and U. Iriso, in Proceedings of the Particle Accelerator Conference, Portland, OR, 2003 (IEEE, Piscataway, NJ, 2003), p. 1801.

[11] H. Hahn, BNL/SNS Technical Note No. 120, 2003.

[12] R. E. Collin, Field Theory of Guided Waves (McGrawHill, New York, 1060), p. 166.

[13] U. Blell, in Proceedings of the 1999 Particle Accelerator Conference, New York, 1999, edited by A. Luccio and W. MacKay (IEEE, Piscataway, NJ, 1999), p. 1727.

[14] D. Davino, J-L. Mi, and N. Tsoupas, BNL/SNS Technical Note No. 112, Rev. 1, 2002. 